Cahiers Charlevoix

Études franco-ontariennes
Cahiers Charlevoix Études franco-ontariennes

or Crevenerix of

\title{
"Le Lynx et le renard ". Un relais déroutant dans la transmission du conte populaire français en Ontario
}

\section{Jean-Pierre Pichette}

Volume 1, 1995

URI : https://id.erudit.org/iderudit/1039463ar

DOI : https://doi.org/10.7202/1039463ar

Aller au sommaire du numéro

\section{Éditeur(s)}

Société Charlevoix

Presses de l’Université d'Ottawa

ISSN

1203-4371 (imprimé)

2371-6878 (numérique)

Découvrir la revue

Citer cet article

Pichette, J.-P. (1995). « Le Lynx et le renard ». Un relais déroutant dans la transmission du conte populaire français en Ontario. Cahiers Charlevoix, 1, 169-240. https://doi.org/10.7202/1039463ar
Résumé de l'article

Son enquête sur " Le Lynx et le renard » amène Jean-Pierre Pichette, ethnologue de l'Université de Sudbury, à la démonstration de l'origine française d'un petit conte animalier recueilli chez les Sauteux du nord de l'Ontario, en dépit de son inculturation amérindienne. Par un curieux retour des choses, l'étudiant parti à la recherche de la culture de l'autre se retrouve plongé dans la sienne propre que le gros bon sens, nourri de préjugés - le mythe du " bon sauvage ", dans sa version écologique contemporaine, joint au complexe du minoritaire -, l'empêche cependant de reconnaître. La privation de contact avec la tradition orale franco-canadienne rendrait ainsi, devant une simple présomption, l'identification impossible et, partant, l'abdication naturelle. 


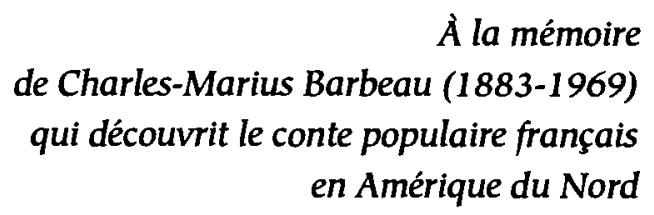

«LE LYNX ET LE RENARD».

UN RELAIS DÉROUTANT DANS LA

TRANSMISSION DU CONTE POPULAIRE

FRANÇAIS EN ONTARIO

Jean-Pierre Pichette

Département de folklore et ethnologie Université de Sudbury, Sudbury

Cahiers Charlevoix 1, 1995, pp. 169-240. 


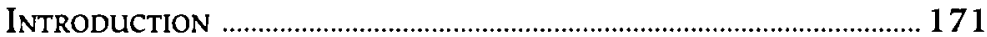

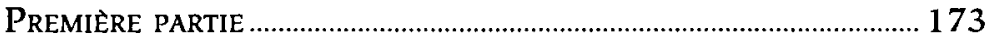

À propos d'un écrit estudiantin récent

À la recherche d'une «légende amérindienne»

DEUXIÈME PARTIE

Un récit amérindien type

A - Connaissance de la vie animale

B - Les Anishinabés

C - Un récit étiologique

$\mathrm{D}$ - Le rituel du contage

E - Autres versions amérindiennes

Conclusion: un récit identitaire

TroISIÈME PARTIE

Des récits parallèles canadiens-français

QUATRIEME PARTIE

Le point tournant

Un premier indice

Un conte bien connu au Canada français

Cinquième PARTIE

L'influence canadienne-française reconnue

Découverte du conte populaire français au Canada

SiXIEME PARTIE

Une tradition française plus ancienne

Une tradition européenne

Conclusion

A - Connaissance de la vie animale

B - Coutume de la pêche sur la glace

C - Récit étiologique

D - Rituel et portée morale

E - Transfert culturel

ÉpILogue

Le chaînon manquant 


\section{«LE LYNX ET LE RENARD» \\ UN RELAIS DÉROUTANT DANS LA \\ TRANSMISSION DU CONTE POPULAIRE \\ FRANÇAIS EN ONTARIO}

\section{INTRODUCTION}

Il est des récits tellement bien adaptés à leur milieu ambiant qu'on peut presque les dire écologiques tellement ils correspondent en tous points à l'environnement physique, à la vie naturelle - végétale et animale - à la vie matérielle, à la vie sociale et à la vie spirituelle du peuple qui les véhicule. Ils sont à ce point investis de la couleur locale que l'idée même qu'ils puissent exister ailleurs dans d'autres cultures paraît souvent une absurdité. C'est d'ailleurs ce que croyaient les premiers assembleurs de contes populaires, les célèbres frères Grimm par exemple ${ }^{1}$.

\footnotetext{
'Voir l'ouvrage d'Ernest Tonnelat, Les Frères Grimm. Leur œuvre de jeunesse, thèse pour le doctorat présentée à la Faculté des lettres de l'Université de Paris, Paris, Librairie Armand Colin, 1912, XII-438 pages; et particulièrement ce passage qui résume la préface à la deuxième édition des Kinder-und Hausmärchen et que Tonnelat considère comme «le premier exposé de la théorie «aryenne»», p. 21 7: «La question de l'origine des contes, jusqu'alors négligée par les frères
} 


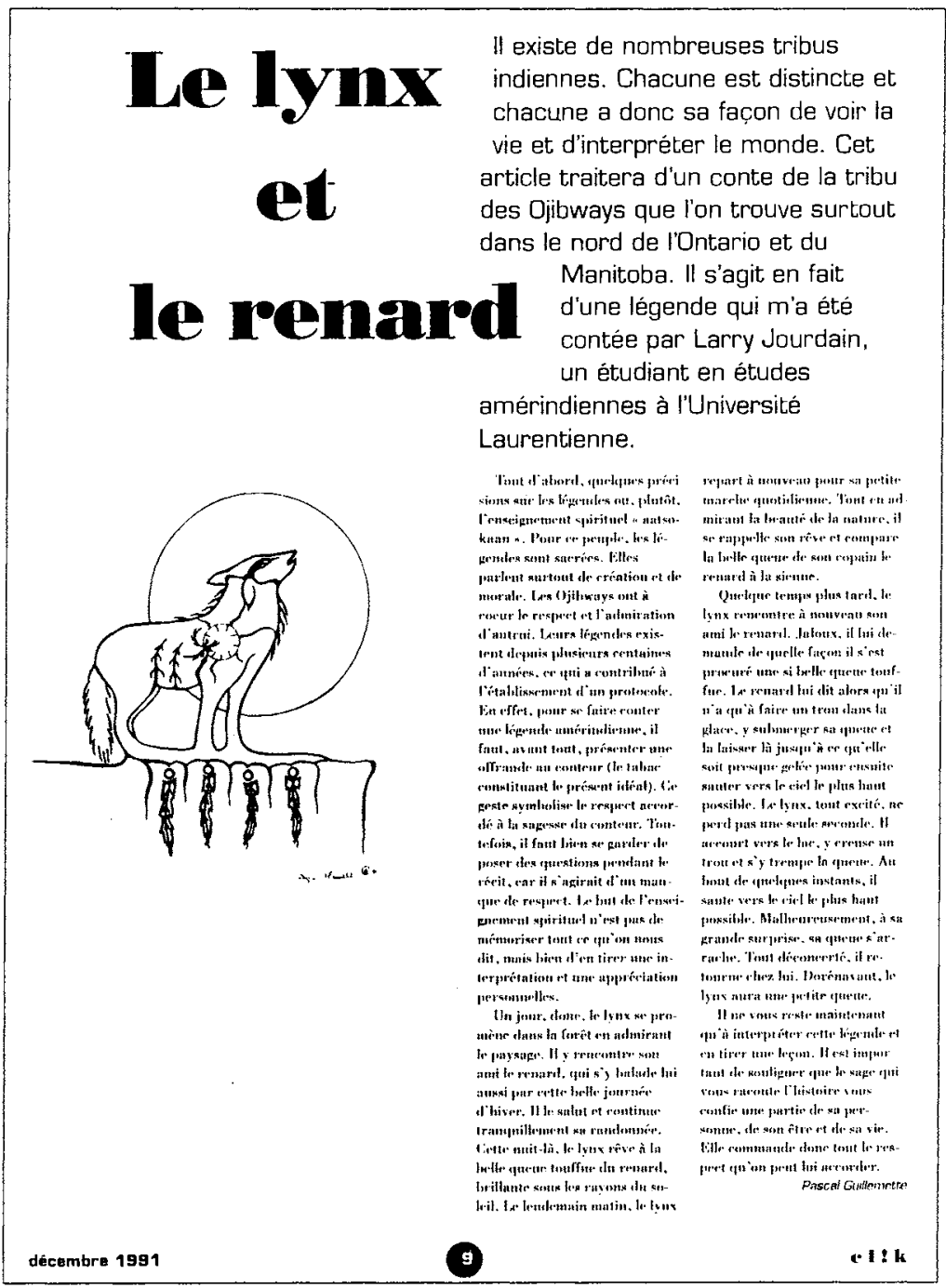


Nous avons récemment découvert, de façon tout à fait fortuite, un tel récit. Nous en proposons l'examen dans les pages qui suivent.

\section{Première partie}

À PROPOS D'UN ÉCRIT ESTUDIANTIN RÉCENT

En décembre 1991, sous la plume de Pascal Guillemette, paraît dans Clik, un magazine destiné aux étudiants du secondaire franco-ontarien ${ }^{2}$, un singulier article intitulé "Le Lynx et le renard", orné d'une illustration de Ken Ense ${ }^{3}$. En un peu moins de cing cents mots, l'auteur nous livre un court récit qu'il s'efforce de situer dans son contexte.

L'introduction compte deux paragraphes. Le premier, composé en gros caractères à la hauteur du titre et sur la largeur de deux colonnes, annonce sans

Grimm, y était enfin abordée. Elle ne pouvait pas comporter pour eux de recherche d'influences étrangères. Fidèles à leur théorie de la poésie populaire spontanée, ils rejetaient l'hypothèse d'un emprunt de province à province allemande. Fleur épique, le conte s'était partout épanoui librement dans l'âme humaine. Toutefois, puisqu'un grand nombre de contes allemands se retrouvaient, identiques, dans les littératures scandinave, anglaise, serbe, hindoue, persane, on pouvait admettre qu'il avait existé, à l'origine, un archétype du conte. C'était le temps où la philologie commençait à démontrer la parenté des langues indo-européennes. Quoi de plus naturel que de faire remonter à une antiquité commune les diverses traditions nationales? Dans une période antérieure à la séparation des peuples, la poésie divine avait été une et simple; depuis, elle avait pris, selon les temps et les lieux, mille apparences diverses; mais elle n'avait pas cessé d'être, en son essence, immuablement fidèle au type fondamental. Le conte, partie intégrante de cette poésie divine, s'était ainsi multiplié en variantes innombrables; mais toutes les variantes avaient un droit égal à se réclamer du conte primitif; aucune d'elles n'était dans la dépendance de sa voisine."

${ }^{2}$ Toronto, décembre $1991, n^{\circ} 27$, p. 9.

${ }^{3}$ De lecture difficile, sa signature a été identifiée par Barry Ace, cousin de l'artiste et chargé de cours en études amérindiennes à l'Université de Sudbury. 
équivoque que le récit donné en titre, «Le Lynx et le renard», provient de la tribu des «Ojibways», d'après sa terminologie anglaise; Sauteux en français ${ }^{4}-$, population «que l'on trouve surtout dans le nord de l'Ontario et du Manitoba», et que son narrateur fut Larry Jourdain, "un étudiant en études amérindiennes à l'Université Laurentienne». Il précise que cette narration est une «légende».

Le second paragraphe d'introduction, qui fait corps avec la suite du texte disposé sur deux colonnes, parle du caractère sacré que ce peuple attribue à ses légendes qui font partie d'un enseignement spirituel dit "aatsokaan» [Aadisokaan qui signifie légende]. Il ajoute que leur narration, au cours des siècles, "a contribué à l'établissement d'un genre de protocole» auquel il faut se soumettre pour obtenir l'approbation du conteur. Ainsi, l'étiquette veut que l'on présente d'abord «une offrande au conteur (le tabac constituant le présent idéal)» puis que l'on écoute attentivement, car «il faut bien se garder de poser des questions pendant le récit, car ceci constitue la marque d'un auditeur irrespectueux", afin d'en «tirer une interprétation» personnelle.

Alors prend place, en deux paragraphes comptant ensemble moins de 200 mots, le récit attendu.

\footnotetext{
${ }^{4}$ Ici, nous suivons la terminologie préconisée par l'équipe du Dictionnaire biographique du Canada ( $D B C$, Québec, les Presses de l'Université Laval, vol. 1, [1966]); elle apparaît au «Glossaire des noms des tribus indiennes» (pp. 12-16) à la suite de l'article "Les Indiens du Nord-Est de l'Amérique» de Jacques Rousseau et George W. Brown: "Les Sauteux étaient divisés en quatre groupes: les Sauteux de la région du lac Supérieur (aujourd'hui parfois appelés en anglais Ojibwas), les Missi[s]saugas de l'île Manitoulin[e] et des rives de la rivière Mississagi, les Outaouais de la baie Georgienne et les Potéouatomis de la rive ouest du lac Huron (dans le Michigan actuel)» (p. 16).
} 
Dans le paragraphe de conclusion, l'auteur de l'article nous invite à «tirer une leçon" de "cette légende», car «le sage» qui l'a racontée à son informateur "commande $[. .$.$] le respect» puisque, avec son histoire, il$ nous "confie, en même temps, une partie de sa personne, de son être et de sa vie».

Auprès de cet article qui occupe la droite de la page, trônant au centre sous le titre, avec lequel il partage la partie gauche de la page, figure une illustration en noir et blanc, de toute évidence à la manière amérindienne des artistes de l'île Manitouline. On y peut voir, sur le bord d'une falaise à laquelle pendent quatre plumes, un renard de profil (qu'on prendrait d'abord pour un loup ou un coyote), la tête relevée, la gueule ouverte, hurlant sans doute à la lune qui l'auréole. Ce dessin en contour montre à l'endroit du cœur de l'animal un cercle en forme de soleil radiant qui éclaire à contre-jour l'avant d'un caribou, dont la tête et le panache débordent dans ce cœur, et à gauche deux arbres, apparemment des pins, qui occupent le flanc droit du renard et sont limités par le tracé des pattes avant et arrière; d'un rayon du soleil, à la hauteur du cou, surgit la silhouette d'un huard qui s'envole et dont la tête forme l'œil apparent du renard.

Le mois suivant, l'Orignal déchaîné, «le journal des étudiants et des étudiantes de l'Université Laurentienne ${ }^{5}$ », reprend le même article, sans son illustration, mais il en modifie le titre: «Le Rêve d'une belle queue. Présentation d'une légende amérindienne». Tout le texte y est distribué sur trois colonnes.

Ici encore, le premier paragraphe reçoit un traitement particulier, la signature de l'auteur le détachant

${ }^{5}$ Sudbury, mardi 14 janvier 1992, vol. 5, no 7 , p. 8. 
du reste du texte. Le deuxième paragraphe de l'introduction de la version Clik y est ensuite fractionné en trois paragraphes. De même le récit, qui ne formait que deux alinéas, en compte cinq à présent. Enfin, la conclusion est à son tour scindée en deux paragraphes. Cependant l'équilibre original de chacune des parties du texte est respecté par l'ajout de trois astérisques séparant l'introduction et le récit proprement dit, et de trois autres astérisques démarquant le récit et la conclusion. À part ces quelques modifications de détail, on ne remarque pas de changement important au texte précédemment paru.

La lecture de l'histoire qui y était rapportée piqua vivement notre curiosité, tout d'abord parce qu'elle relevait d'une tradition orale avec laquelle nous étions trop peu familier et, ensuite, parce que le jeune journaliste qui l'avait consignée avait fait d'une certaine façon, en la recueillant, œuvre ethnologique.

Peu après, l'occasion nous fut donnée de rencontrer et de féliciter cet étudiant avec qui nous discutâmes du document qu'il venait de publier, nous attardant surtout à des points qu'il n'avait pas cru bon de noter ou de demander, en particulier à propos de l'origine de cette tradition. Il parut apprécier le fait qu'un professeur ait lu son modeste article avec autant d'intérêt, mais il se montra par la suite étonné et peut-être quelque peu troublé par la nature de ces questions qu'il n'avait en aucun cas prévues et qui étaient de toute façon en dehors de ses préoccupations au moment de produire cet article. Néanmoins, cet entretien bref et cordial s'acheva sur l'offre qu'il nous fit, après que nous lui eûmes fait observer que les deux versions publiées divergeaient légèrement par le titre et par la forme, de nous remettre un 
exemplaire de son texte manuscrit. Il s'exécuta fidèlement, mais nous n'y trouvâmes pas les éléments vraiment nouveaux que nous espérions y découvrir. Les autres discussions que nous eûmes à cette époque n'apportèrent, elles non plus, aucun fait significatif à ce dossier.

\section{À LA RECHERCHE D'UNE «LÉGENDE AMERINDIENNE»}

Récemment ${ }^{6}$, environ deux ans et demi plus tard, nous avons pu reprendre entièrement cette conversation à propos de l'article en cause afin de bien saisir les circonstances qui avaient amené son auteur à l'écrire.

Pascal Guillemette était, au cours de l'année 1991 1992, en deuxième année de philosophie à l'université Laurentienne et agissait régulièrement comme journaliste au journal français des étudiants de cette institution. C'est ainsi que la direction du magazine Clik, qui opérait de Toronto, sollicita un jour sa collaboration au numéro thématique qu'elle préparait sur les autochtones. Sa mission: retrouver une «légende amérindienne» dans le nord de l'Ontario où la chose semblait apparemment plus facile.

Mais pour l'étudiant Guillemette né à Laval (Québec), élevé à Dartmouth (Nouvelle-Écosse) et à Burlington (Ontario), installé depuis deux ans à Sudbury pour ses études universitaires, la tâche n'était pas si simple: il ne connaissait aucun Amérindien ni, encore moins, aucune légende autochtone. Débrouillard, il entre aussitôt en contact avec un professeur du département d'études amérindiennes de l'Université de Sudbury, monsieur Roger Spielman, le priant de lui raconter tout

\footnotetext{
${ }^{6}$ Entrevue réalisée à Sudbury le 12 mai 1994, 6 p. ms.
} 
bonnement un tel récit qui pourrait satisfaire l'éditeur torontois. L'étudiant a frappé à la bonne porte: le professeur s'intéresse à la littérature orale amérindienne, il a recueilli personnellement et connaît certes de nombreux récits qui auraient tôt fait de contenter son interlocuteur, mais il lui explique que, n'étant pas amérindien lui-même, il poserait un geste "irrespectueux» envers la tradition orale d'origine s'il se constituait narrateur. Cependant, désireux d'aider l'étudiant, il lui fixe rendez-vous pour le lendemain, s'engageant à lui présenter un étudiant autochtone qui devrait pouvoir raconter le récit recherché.

Le lendemain, le jeune journaliste rencontre l'étudiant annoncé. Il s'agit de Larry Jourdain, jeune homme d'environ 23 ans de la tribu des Sauteux de la région de Fort-Frances, dans le nord-ouest de l'Ontario. L'entrevue se déroule en anglais dans le bureau du professeur et en sa présence. Après les présentations d'usage, l'enquêteur expose le but de sa recherche et demande au jeune Amérindien de lui raconter «une légende représentative de sa culture». Celui-ci accepte et lui fait le récit suivant dont l'enregistrement, exécuté uniquement par mesure de précaution et pour des fins journalistiques, n'a malheureusement pas été conservé.

\section{[Le Lynx et le renard] ${ }^{7}$}

[Un jour, donc, le] Le lynx se promène dans la forêt en admirant le paysage. Il [y rencontre son] voit soudaine-

\footnotetext{
${ }^{7}$ Le texte ci-dessous suit la version manuscrite de Pascal Guillemette, expurgée de quelques coquilles; les varlantes de la version Clik apparaissent en italiques entre crochets ([possible]) et celles de l'Orignal déchaîné figurent en gras entre parenthèses (salue). L'enquêteur nous a confié que son informateur, qui s'exprimait en anglais, utllisait les mots lynx et fox pour désigner les protagonistes de son récit.
} 
ment son ami le renard qui [s'y balade aussi par cette belle journée d'hiver], par cette belle journée d'hiver, se balade aussi. Le lynx le salua (salue) tout simplement et continua sa randonnée [Il le salue et continue tranquillement sa randonnée]. (alinéa) Cette même nuit (La nuit venue), le lynx rêve à la belle queue touffue et bien garnie du renard, queue qui brillait aux rayons du soleil [Cette nuit-ld, le lynx rêve à la belle queue touffue du renard, brillante sous les rayons du soleil ]. (alinéa) Le lendemain matin, le lynx repart à nouveau pour sa petite marche quotidienne. Tout en admirant la beauté de la nature, il se rappelle le rêve qu'il a fait la veille [son rêve] et compare la belle queue de son copain le renard à la sienne.

Quelque temps plus tard, le lynx rencontre à nouveau son ami le renard. Jaloux, il lui demande de quelle façon il s'est procuré une si belle queue touffue. Le renard lui dit alors qu'il n'a qu'à faire un trou dans la glace, y submerger sa queue et la laisser là jusqu'à ce qu'elle soit presque gelée pour ensuite sauter vers le ciel le plus haut possible. (alinéa) Le lynx, tout excité, ne perd pas une seule seconde. Il accourt au [vers le] lac, y creuse un trou et s'y trempe la queue. Au bout de quelques instants, il saute vers le ciel le plus haut qu'il peut [possible]. Cependant [Malheureusement], à sa grande surprise, sa queue s'arrache. Tout déconcerté, il retourne chez lui. Dorénavant, le lynx aura une petite queue courte [une petite queue].

À la suite de cette narration, le jeune journaliste apprend qu'il s'agit là d'une véritable «légende» de la tribu des Sauteux, que son informateur la tient de l'un "de ses grands-pères» et qu'on se l'est ainsi transmise par «tradition orale» «de génération en génération ${ }^{8}$ ». À la demande de Pascal Guillemette, qui, en outre, désire en connaître le rituel en usage

\footnotetext{
${ }^{8}$ Monsieur Roger Spielman a bien voulu rechercher pour nous les renseignements suivants qu'il nous a communiqués le 20 juin 1994: Larry Jourdain aurait appris son récit en février 1983 de Bob Kizhig (décédé en 1993), surnommé "Wezhiobines» [Aigle endimanché], au lac Lacroix, Ontario; intitulée en anglais «How the Lynx Got a Short Tail», cette «légende» sert à transmettre un enseignement moral.
} 
dans la culture traditionnelle de cette bande amérindienne, le jeune conteur explique le «protocole» narratif qui sera présenté, comme nous l'avons déjà vu, dans l'article à l'étude. Le jeune correspondant confirmera plus tard que, considérant qu'il avait alors obtenu tout le matériel nécessaire à son projet, la rencontre prit fin sans qu'il soit question d'autres récits: «'étais très ouvert à [tout ce qu'il pouvait me raconter]; d'ailleurs, je [ne] connaissais pas du tout la culture objibouée. Donc, j'ai pris ce qu'on m'a donné.» Satisfait, il rédigea son article et le publia, plutôt deux fois qu'une. Travail bien fait. Mission accomplie.

Comment en effet mieux approcher une culture que par la tradition des anciens, pieusement recueillie et conservée dans les mémoires, porteuse d'une sagesse séculaire, sinon millénaire, celle qui a pénétré les jeunes esprits d'un peuple par les mots de leur langue maternelle, celle qui a ainsi façonné leurs pensées les plus intimes, celle qui leur parle d'euxmêmes, de la vie des leurs et de leurs coutumes, de leur terre et de la nature qui les environne?

\section{Deuxtème partie}

\section{UN RECIT AMERINDIEN TYPE}

À l'observateur que nous sommes, voilà, condensée dans cette humble narration, toute l'essence d'une culture autochtone si différente de la nôtre: son rapport évident aux mythes des origines et aux récits du héros mystificateur, sa connaissance de la nature et de la vie animale, joints au rituel même de la narration et à l'enseignement spirituel qu'elle véhicule, nous ramènent aux lieux communs les plus remarqués 
dans les traditions orales amérindiennes.

\section{A - Connaissance de la Vie animale}

La connaissance certaine, bien que rudimentaire, de la vie animale est apparente dans cette brève histoire dont elle contribue, par le fait même, à révéler un premier niveau d'enseignement strictement littéral.

Les acteurs de cette histoire, le lynx ou loup-cervier et le renard, sont des mammifères bien connus de la faune nord-américaine. La simple mention de leurs noms fournit alors à l'auditeur un contexte ou une série de présupposés que le conteur n'a pas à donner expressément à des adultes ou à des gens qui vivent près des forêts, mais qu'il pourrait éventuellement décrire à des étrangers ou à des personnes peu familières avec la vie animale. Au rang de ces éléments supposés, ou non exposés par le narrateur, il importe de traiter brièvement des trois suivants: la description de ces animaux, leurs mœurs et leur habitat.

Le renard ${ }^{9}$, qui est plus petit de taille que le loupcervier, compte quatre espèces canadiennes: le renard arctique (Alopex lagopus), le renard roux (Vulpes vulpes), le renard véloce (Vulpes velox) et le renard gris (Urocyon cinereoargenteus), et une quinzaine de sous-espèces. De toutes celles-ci, c'est le renard roux qui est l'espèce la plus commune avec ses neuf sousespèces répandues à la grandeur du territoire canadien. Cet animal a l'allure d'un «petit chien élancé, ayant la face et les oreilles pointues, le pelage long et

\footnotetext{
${ }^{9}$ Les renseignements de cette partie sont tirés de l'ouvrage d'A.W.F. Banfield, Les Mammifères du Canada, deuxième édition, Sainte-Foy, Les Presses de l'Université Laval, 1977, pp. 275-283.
} 
soyeux et la queue touffue». Élément significatif pour notre propos, son appendice caudal fait à peu près la moitié de la longueur de son corps. Timide et nerveux, chasseur solitaire durant tout l'automne et l'hiver, ce prédateur jouit d'un flair très subtil et d'une ouïe particulièrement fine. Il est nomade et n'hésite pas à occuper le terrier de la marmotte ou à creuser le sien au besoin. Plutôt nocturne ou matinal, il se nourrit de tout ce qu'il peut trouver: de viande surtout en hiver, petits mammifères qu'il peut flairer et débusquer même sous la neige et oiseaux, et l'été de végétaux, d'invertébrés et de vertébrés, les poissons inclus. Prévoyant, il sait faire des provisions pour l'hiver. Le renard est la proie du lynx, dont il peut cependant à l'occasion manger les petits, mais son agilité lui permet de se placer hors d'atteinte de son ennemi le loup. On le retrouve à la lisière des forêts, dans les clairières, les champs et sur les rives des lacs.

Le lyn $x^{10}$ a des membres plus longs que le renard et son poids double celui de ce dernier; mais sa queue tronquée ne mesure qu'une dizaine de centimètres (de quatre à cinq pouces). On en compte deux espèces au Canada: le loup-cervier (Lynx), aussi appelé lynx du Canada, et le lynx roux (Lynx rufus) qui se ramifient en sept sous-espèces; le premier occupe les forêts du Nord à la grandeur du pays, le second se concentrant le long de la frontière sud, de la Nouvelle-Écosse à la Colombie-Britannique. On rencontre les deux en Ontario, mais le loup-cervier y serait le plus commun. Selon Henri de Puyjalon, le loup-cervier «se sépare des chats, dont il est néanmoins proche parent, par la grandeur de ses pattes, par ses oreilles garnies de pinceaux et par la peti-

${ }^{10}$ Banfield, op. cit., pp. 325-331. 
tesse de sa queue qui se réduit à un appendice de quelques centimètres de longueur, à peine visible au milieu de la fourrure qui embellit et protège les parties voisines ${ }^{11}$ ». Comme le renard, c'est un solitaire qui circule tout l'hiver et chasse de la fin de la nuit jusqu'au matin. Il se nourrit principalement de lièvres, de rats musqués, de souris, d'écureuils, de renards et de mouffettes, mais il n'hésite pas à s'attaquer aux caribous et aux cerfs, d'où son épithète de cervier. "Au repos, il se lisse le poil.» Une ouie et une vue très aiguisées - ne dit-on pas avoir un œil de lynx? - compensent son flair médiocre. Très agile, il peut facilement se protéger en grimpant aux arbres sous les branches desquels il fait ordinairement son abri. Le loup-cervier préfère les forêts boréales, mais il s'aventure parfois dans des terrains découverts.

La comparaison de ces deux mammifères permet donc de dégager les traits suivants qui nous serviront dans la suite de notre analyse. Deux caractéristiques anatomiques sont particulièrement évidentes: d'une part, la supériorité physique du loup-cervier, manifestée par sa force et sa taille, en fait un prédateur du renard; d'autre part, la longue queue touffue de ce dernier contraste avec le moignon du lynx.

Au plan éthologique, une nette différence apparaît encore qui teinte peut-être toujours un peu l'image qu'on se fait de l'un et de l'autre: elle concerne la qualité de leur flair. À se fier à l'observateur privilégié de la faune canadienne au XIX ${ }^{e}$ siècle, et de ces deux mammifères en particulier, que fut Henri de Puyjalon, c'est par ce trait qu'on semble juger de la finesse du renard: «Si sa finesse est très grande,

11 Guide du chasseur de pelleterie, Montréal, Pierre J. Bédard, 1893, p. 65. 
écrit-il, sa défiance est extrême. Son odorat tient du merveilleux ${ }^{12}$ ». Ailleurs, il renchérit en le disant «doué d'un odorat si développé qu'il évente l'homme le moins parfumé d'une lieue ${ }^{13}$." Quant au loup-cervier, dont le flair est médiocre, de Puyjalon avance ce jugement: «Si l'on établissait une échelle de la valeur comparative des facultés intellectuelles ou instinctives que possède chaque animal, on pourrait classer le loup-cervier très près du dernier échelon. Son instinct semble peu développé ${ }^{14}$.» Plus tard, il ajoutera cette nuance: "C'est un animal peu intelligent, en apparence ${ }^{15} . "$

Quant au plan écologique, on comprend que le renard vivant à la lisière de la forêt et le lynx préférant le cœur des forêts, ils ont tendance à s'éviter, mais qu'ils peuvent également se rencontrer. En effet, leurs habitudes de chasse en solitaire, de nuit surtout, les amènent à convoiter occasionnellement les mêmes proies.

En résumé, on peut dire que ce sont des ennemis naturels et qu'ils se comportent véritablement, selon le dicton populaire, "comme chien et chat", ce qu'ils sont d'ailleurs, le renard appartenant à la famille des canidés et le loup-cervier à la famille des félidés.

\section{B - Les ANISHINABES}

Le conteur de cette histoire, nous l'avons vu précédemment, est un jeune homme de la tribu des Sauteux du nord de l'Ontario.

\footnotetext{
${ }^{12}$ Histoire naturelle d l'usage des chasseurs canadiens et des éleveurs d'animaux d fourrure, Québec, Le «Soleil», 1900, p. 160.

${ }^{13}$ Guide du chasseur de pelleterie, op. cit., p. 35.

${ }^{14}$ Ibid., p. 64.

${ }^{15}$ Histoire naturelle, op. cit., p. 149.
} 
Après avoir quitté la côte atlantique il y a plus de 500 ans, ce groupe algique, rattaché par la langue aux Outaouais et aux Potéouatomis (famille algonquine), s'est installé dans la région du SaultSainte-Marie en Ontario, d'où leur nom de Sauteux, et de là s'est disséminé tout autour de la région des Grands Lacs. On les retrouve aujourd'hui dans trois provinces canadiennes - l'Ontario, le Manitoba et la Saskatchewan - et dans cinq États du nord des États-Unis - le Michigan, le Wisconsin, le Minnesota, le Dakota du Nord et le Montana - où ils sont répartis dans une centaine de réserves et dans des grandes villes - Toronto (Ontario) et Winnipeg (Manitoba); Grand Rapids (Michigan) et Minneapolis (Minnesota). Ils forment une population totale de 200000 habitants dont les deux tiers résident toujours en territoire canadien ${ }^{16}$. D'abord appelés Saulteurs par les missionnaires jésuites ${ }^{17}$, ils reçurent d'autres noms par la suite: Sautereaux, Chippeouais, Mississauga, Ojibwa, Odjibways, Ojiboués ou autres; beaucoup d'entre eux se désignent maintenant par l'appellation Anishinabés ${ }^{18}$.

Ces nomades, qui se déplaçaient selon les saisons sur leurs terrains de chasse, de pêche, de cueillette (baies et riz sauvage, eau d'érable), utilisaient abondamment l'écorce de bouleau pour fabriquer leurs habitations, leurs canots et de nombreux paniers et autres contenants. Ils cultivaient aussi le

${ }^{16}$ Helen Hornbeck Tanner, The Ojibwa, New-York et Philadelphie, Chelsea House Publishers, "Indians of North America», [c1992], pp. 14-19.

${ }^{17}$ Ibid., p. 34. Voir le "Glossaire des noms des tribus indiennes», op. cit., ( $D B C$, vol. 1), p. 16.

${ }^{18}$ Tanner, op. cit., p. 14. 
maïs, la citrouille et la courge. Notons, pour notre propos, qu'ils pratiquaient la pêche sur la glace ${ }^{19}$.

On le voit, ce groupe occupe le territoire canadien et connaît très bien la faune qu'il contient. Rien done dans l'histoire du Lynx et du renard qui lui soit autrement que familier.

\section{C - UN RÉCIT ÉTIOLOGIQUE}

En outre, ce récit s'apparente aux mythes amérindiens qui composent cette "histoire sainte» des peuples primitifs qui explique l'origine et l'organisation de l'univers, des êtres et des choses. Dans ce contexte, l'histoire du lynx, animal stupide, qui perdit sa queue en suivant le conseil trompeur du matois renard trouve logiquement sa place. À l'origine, dans un monde antérieur au nôtre, les deux mammifères auraient été des amis et le lynx pourvu d'une queue plus longue que de nos jours, quoique moins touffue que celle du renard. Telle est bien la situation initiale de notre récit. C'est l'envie du lynx jointe à sa stupidité qui expliquent la petitesse de sa queue et aussi l'inimitié des deux animaux qui sévit dans le monde actuel.

${ }^{19}$ Ibid., pp. 21-24. Jacques Rousseau et George W. Brown font les mêmes observations dans "Les Indiens du Nord-Est de l'Amérique», Dictionnaire biographique du Canada, vol. 1, [Québec], Les Presses de l'Université Laval, [1966], p. 6. D'autre part, Pierre Boucher, dans son Histoire véritable et naturelle des mœurs et productions du pays de la Nouvelle-France vulgairement dite le Canada (À Paris, chez Florentin Lambert, 1664, [23]-168 pages; Boucherville, Société historique de Boucherville, 1964, LXIV-[23]-415 pages), rapporte ce témoignage au chapitre deuxième (p. 26), en parlant de la beauté «du pays des Hurons», "situé sur le bord du grand Lac»: "l'y ay veu une pesche qui est fort agreable, qui se fait aussi-bien l'Hyver sous les glaces, que pendant l'Esté; c'est celle du Haran [hareng de lac: Coregonus artedii] dont il y a abondance." 
Bien plus, la seule présence de l'animal trompeur - ici le renard qui mystifie complètement le lynx renvoie, entre autres, à la mythologie amérindienne. On sait, principalement par l'étude que Paul Radin lui a consacrée $e^{20}$, combien le cycle du Fripon est général chez les autochtones d'Amérique qui le tiennent habituellement pour une histoire sacrée.

\section{D - LE RITUEL DU CONTAGE}

L'expérience que rapporte Paul Radin dans son livre le Fripon divin offre, au plan du rituel, des similitudes frappantes avec le cas présentement à l'étude. C'est un «Winnebago pur sang», Sam Blowsnake qui lui servit d'intermédiaire dans la cueillette du mythe du Fripon, car, s'agissant "d'un mythe sacré» et l'auteur étant "un étranger, par surcroît un blanc», il lui aurait été extrêmement difficile d'en obtenir le récit. D'ailleurs, Blowsnake lui-même ne put l'obtenir de son propre père qui connaissait bien ce mythe, mais qui, s'étant converti à une autre religion, ne remplissait plus les conditions essentielles pour le raconter: y croire et en être autorisé par la tradition ${ }^{21}$. Radin explique que le «droit de raconter un mythe, une waikầ - c'est-à-dire une histoire sacrée, par opposition à une histoire profane appelée worak «appartient chez les Winnebagos, soit à une famille déterminée, soit à un personnage déterminé» et que "ce droit est, en un certain sens, sa "propriété" et [qu']il a, de ce fait, une valeur marchande considérable $^{22} »$. De plus, il est important que le mythe soit

\footnotetext{
${ }^{20}$ Clarl] G[ustav] Jung, Karl Kerényi et Paul Radin, Le Fripon divin. Un mythe indien, [Préface de Paul Radin, 1954], traduction de Arthur Reiss, Genève, Librairie de l'Université Georg, [1984], 205 pages.

21 Ibid., pp. 89-90.

22 Ibid., p. 100.
} 
transmis selon «les circonstances appropriées». C'est ce que vérifia Radin: «Par "circonstances appropriées", j'entends les dons de tabac adéquats et les cadeaux conformes à la valeur traditionnelle du mythe, qui furent offerts au narrateur ${ }^{23}$." Plus loin, il ajoute qu'il "était interdit de raconter les waikâ en été ${ }^{24}$ ». Ces règles, vraies pour la tradition littéraire des Winnebagos, paraissent également communes à la plupart des tribus amérindiennes. Dans l'introduction et la conclusion de son article, Pascal Guillemette avait, lui aussi, noté, pour la tradition des Sauteux du nord de l'Ontario, un tel protocole narratif comportant essentiellement une offrande de tabac au conteur; ce rite, à la fois propitiatoire et rémunératoire, était destiné à acheter la sagesse du conteur tout en manifestant la valeur qu'on y accorde.

\section{E - AUTRES VERSIONS AMÉRINDIENNES}

En plus de posséder les attributs de la culture traditionnelle des Sauteux, ce récit est attesté ailleurs dans la famille algique. Notre collègue Roger Spielman en a recueilli, à la réserve de Pikogan, dans le nordouest du Québec, une version très rapprochée de celle de notre jeune journaliste. Étant donné sa brièveté, nous la présentons ci-après in extenso dans sa traduction française.

Le Lynx et le renard [traduction]

[Voici une autre histoire à propos du lynx.] /

Le lynx rencontra le renard / et lui demanda: «Comment astu obtenu une aussi belle queue?» / Le renard répondit: "Je fais un trou dans la glace, / je mets ma queue dans le trou /

${ }^{23}$ Ibid., p. 90.

${ }^{24}$ Ibid., p. 98. 
puis, quand elle est gelée, je me relève vivement." / C'est ce que le renard lui dit. I

Quelque temps après, le lynx l'essaya d̀ son tour. Il creusa un trou dans la glace et plongea sa queue dans le trou. / Après un certain temps, sa queue gela [dans le trou]. / Alors [quand sa queue fut gelée], il se releva si rapidement / que sa queue se cassa net. / Sa queue se détacha quand il se releva. / C'est pourquoi le lynx a [aujourd'hui] une queue courte ${ }^{25}$.

Par ailleurs, Juliette Gauthier (1888-1972), qui fit carrière d'interprète de chansons traditionnelles canadiennes-françaises et autochtones sous le nom de Juliette Gaultier de la Vérendrye, rédigea au début des années 1940 un manuscrit resté longtemps inédit, «Around the Birch Bark Wigwam» (Autour du wigwam en écorce de bouleau), tiré de la cueillette ethnographique qu'elle avait faite chez les Algonquins de la vallée de la haute Gatineau. Récemment des chercheurs du Musée canadien des civilisations ont publié un choix de textes de cette compilation dans lequel se trouve une version du récit qui nous occupe $^{26}$ et que nous citons intégralement:

\section{Canulah, le renard et l'ours}

C'était au printemps, à la fonte des neiges. Canulah pêchait sur la glace. Il avait pris du poisson, l'avait placé sur son traîneau et revenait chez lui. Un renard traversa sa piste un

\footnotetext{
${ }^{25}$ Version d'Albert Mowatt, Algonquin de la réserve Pikogan au Québec, recueillie durant l'hiver 1983 par Roger et Ruth Spielman. Ce récit, enregistré en algonquin, apparaît en transcription dans sa langue originale et en traduction anglaise dans la collection inédite «Algonquin Text Archives. Texts collected by Roger and Ruth Spielman, Pikogan (P.Q.) 1980-1990»; c'est le n' 43 intitulé "The Lynx and the Fox". Version aimablement communiquée par Monsieur Spielman, professeur à l'Université de Sudbury, le 17 mai 1994.

${ }^{26}$ Daniel Clément et Noëline Martín, "Coutumes et légendes algonquines, d'après un inédit de Juliette Gaultier de la Vérendrye», dans Recherches amérindiennes au Québec, Montréal, vol. 23, $\mathrm{n}^{\text {os }} 2-3$, "Les Algonquins", automne 1993, pp. 69-85.
} 
peu en avant de lui. Le renard se coucha par terre et fit semblant d'être mort. Au passage, le pêcheur le ramassa par la queue et le mit dans son traîneau.

Mais le renard reprit connaissance et repéra le poisson. Il sauta hors du traîneau et s'enfuit vers le bois en emportant tout avec lui. Chemin faisant, il rencontra un ours. En voyant le renard avec du poisson, l'ours s'enquit où il l'avait pris. "Je me suis seulement assis près d'un trou sur la glace, dit le renard, et quand le poisson mordillait ma queue, je sautais rapidement de côté et le poisson restait accroché."

Alors, l'ours crut qu'il pouvait faire de même. Il s'assit donc sur la glace et mit sa queue d l'eau. Il resta toutefois assis trop longtemps et sa queue gelée resta sur place. Depuis ce temps, tous les ours naissent avec de courtes queues.

"Autrefois et dans un autre monde, ajoute Canulah (Nadawesi-Iroquois Bird), tous les ours avaient des queues grosses et longues."

(Légende des Indiens Bush-Men [aussi appelés Têtes-deBoule] $)^{27}$.

Malgré quelques divergences, il s'agit bien ici de notre histoire de «la pêche à la queue», précédée cependant d'un nouvel épisode, «le vol du poisson», où le renard trompe le pêcheur-narrateur Canulah en simulant la mort pour lui dérober son poisson. L'adversaire du renard n'est plus un lynx qui désire, par vanité, obtenir une aussi belle queue que la sienne, mais un ours famélique qui veut se procurer du poisson. Le mode d'emploi est le même - immerger sa queue dans l'eau glacée - et la conclusion étiologique similaire - perte de l'appendice caudal qui explique que «depuis ce temps, tous les ours naissent avec de courtes queues», car auparavant "et dans un autre monde, [...] tous les ours avaient des queues grosses et longues».

${ }^{27}$ Ibid, p. 82. 
La diffusion de ce récit dans les populations amérindiennes est certainement plus grande que ne peuvent le faire voir les trois versions utilisées ici. Déjà, en 1946, Stith Thompson en rapportait treize versions dans un tableau de son remarquable traité sur le conte populaire ${ }^{28}$. De nos jours, leur nombre devrait être encore plus important.

\section{CONCLUSION: UN RÉCIT IDENTITAIRE}

Par la connaissance qu'il montre de la vie animale, par le conteur sauteux qui l'a transmis, par son caractère étiologique, par le rituel qui l'encadre et par sa diffusion à l'intérieur du territoire algique, ce conte manifeste de façon indubitable une allure tout à fait indigène et paraît à coup sûr auréolé des attributs d'un récit identitaire. La perception de cette personnalité empreinte de couleur locale, issue du mariage si intimement réussi entre la nature environnante et la culture matérielle, sociale et spirituelle des peuples algiques, se vérifie par le contexte de narration du récit à l'étude: à un journaliste en herbe qui recherche "une légende typiquement amérindienne», le jeune narrateur sauteux n'hésite aucunement à raconter «Le Lynx et le renard», qu'il tient de source orale d'un de ses aïeuls. De là à affirmer que ce récit a été créé par une imagination amérindienne dont les descendants auraient la jouissance et la propriété exclusive, il n'y a qu'un pas.

\footnotetext{
${ }^{28}$ The Folktale, Berkeley et Los Angeles, University of California Press, [1946; réimpression en 1977], p. 288. Toutefois, son anthologie, Tales of the North American Indians (Bloomington, Indiana University Press, [1929], «Midland Book» MB-91, [1966]), en présente une version sauteuse: "The Fox and the Wolf» [Le Renard et le loup], pp. 254-257, surtout le passage des pp. 256-257.
} 


\section{Troisième PARTIE}

DES RÉCTTS PARALLĖLES CANADIENS-FRANÇAIS

Qui a le moindrement fréquenté la tradition orale tient toujours l'exclusivité pour suspecte étant donné que les récits qu'elle véhicule ont le plus souvent une portée universelle. Depuis le milieu du XIX ${ }^{e}$ siècle, on les a en effet repérés dans des régions fort éloignées les unes des autres, tant géographiquement que culturellement. Même si peu de barrières peuvent leur résister, il n'en demeure pas moins qu'ils conservent souvent des traces muettes de ces transmissions: soumis à une analyse minutieuse, éléments, traits, épisodes, thèmes livrent alors leur témoignage et permettent d'induire des états antérieurs qui n'ont pas toujours été attestés. C'est ainsi qu'on a pu prouver qu'un conte populaire, bien connu au Canada français, provenait de la France même si aucune version n'y avait encore été recueillie ${ }^{29}$.

En effet, l'exclusivité doit dès maintenant souffrir un premier accroc puisque la présente histoire n'est pas sans nous rappeler une histoire semblable mettant en scène un renard qui enseigne à un loup comment pêcher de la truite avec sa queue. Nous la reprenons ici pour fins de comparaison.

Pourquoi les loups ont la queue courte

Un jour, un renard et un loup se trouvaient ensemble. Le renard avait invité le loup pour souper. Il avait de la bonne truite. I[l] dit:

\footnotetext{
${ }^{29}$ C'est une des conclusions de Margaret Low dans sa remarquable thèse de doctorat encore inédite, "L'Oiseau mystérieux du château volant. Monographie internationale du conte type Aa.-Th. $708 \mathrm{~A}^{*}$ (462)", Thèse de doctorat, Québec, Université Laval, 1978, XXVI-473 pages.
} 
- Veux-tu me dire où [est]-ce que tu prends ta truite? T'as toujours de la bonne truite, moi je peux pas en trouver.

- Je la pêche, je la pêche.

- Comment [est]-ce [que] tu pêches fa?

- Je pêche ça dans un trou dans la glace. Je pêche fa avec ma queue.

- Veux-tu me montrer comment faire?

- Viens demain matin, je te montrerai.

Le lendemain matin, il faisait un froid sibérien. Le loup et le renard se rendent à la rivière $p$ [u] is le renard se faisait aider du loup à creuser un trou dans la glace. Quand le trou a été grand assez, le renard dit au loup:

- Mets ta queue dedans plu]is quand tu sentiras que ca tire - ca peut prendre un peu de temps - tu donneras un coup sec plu]is tu en auras une bonne charge.

Toujours, le loup met sa queue dans la rivière p[u]is attend, p[u]is attend. Le renard rôdait autour p[u]is ill] demande:

- Quoi, en as-tu pas encore une charge?

- Non, ça tire pas.

- Attends encore une heure.

III] attend, attend, attend. Le loup dit au renard:

- Je crois [que] j'en ai une bonne charge parce que je sens que ça tire.

- La, donne un bon coup sec, tu vas en avoir une bonne.

Là, ill] donne un bon coup p[u]is la queue [lu]i casse. Depuis ce temps-là, les loups ont la queue courte ${ }^{30}$.

Ce récit appartient à la collection acadienne de Catherine Jolicœur qui le recueillit en 1974 sur bande

${ }^{30} \mathrm{CEA}$, collection Catherine Jollcœur, AF-142, enreg. 1992. Cité dans l'Acadie des Maritimes, études thématiques des débuts a nos jours, sous la direction de Jean Daigle, Moncton, Chaire d'études acadiennes, Université de Moncton, 1993, p. 687. 
magnétique de Mme Odile Desrosiers, 61 ans, de Robertville au Nouveau-Brunswick.

On reconnaît bien la même histoire que dans les versions amérindiennes de départ, avec toutefois les quelques variantes que voici: au lieu d'envier la belle queue du renard, c'est plutôt sa façon de prendre du poisson qui séduit l'animal dupé comme dans le récit de Canulah; ce dernier rôle, joué tantôt par un lynx, ou un ours, l'est maintenant par un loup. La mystification demeure néanmoins la même: immobiliser sa queue dans un trou pratiqué à travers la glace jusqu'à ce qu'elle gèle complètement d'abord, puis qu'elle casse enfin en s'efforçant de l'en retirer; ce qui fait que la conclusion demeure inchangée: «Depuis ce temps-là, les loups ont la queue courte.» Cette constatation mériterait d'être nuancée, car le loup n'a pas la queue courte ${ }^{31}$, mais une queue moins touffue et plus courte que le renard.

L'explication étiologique s'avère parfois un élément accessoire qui n'est pas toujours présent dans la tradition ${ }^{32}$, comme en fait foi la version francoaméricaine qui suit.

\section{Gros-Loup et Tit-Renard}

Bon bien, une fois c'était Gros-Loup puis Tit-Renard. Puis

\footnotetext{
${ }^{31}$ Elle mesure de 36 à 50 centimètres d'après Banfield, op. cit., p. 271 . ${ }^{32}$ Marie-Louise Tenèze, citant les subdivisions établies par Oskar Daehnhardt, rappelle cette distinction entre légendes essentiellement étiologiques, "soit celles qui sont fondamentalement construites en vue de l'explication à fournir", et légendes accidentellement étiologiques, "celles où une telle fin se surajoute à une narration existant parfaitement en et pour elle-même»; cf. Paul Delarue et Marie-Louise Tenèze, Le Conte populaire français, Catalogue raisonné des versions de France et des pays de langue française d'outre-mer: Canada, Louisiane, Ilôts [sic] français des États-Unis, Antilles Françaises, Haiti, Ile Maurice, La Réunion, Tome troisième, Paris, Éditions G.-P. Maisonneuve et Larose, [1976], p. 8.
} 
Gros-Loup, c'était un moine [joueur de tours], ça. Il dit d Tit-Renard:

- Viens-tu, on va aller d la pêche?

Tit-Renard, il dit:

- C'est bien correct.

[Ça] fait que, rendus sur le lac, c'est en hiver, [ca] fait qu'ils font un trou, défoncent la glace. Puis Gros-Loup, il dit à TitRenard:

- Mets ta queue dedans là.

[Ça] fait que Tit-Renard met sa queue dedans puis GrosLoup commence:

- Gèle, la queue; gèle, la queue; gèle, la queue.

Puis tout d'un coup, il dit a Tit-Renard:

- Bien, il dit, donne un coup ld.

Tit-Renard donne un coup et puis la queue lui reste dans le trou ld. Elle était pris[e] dans la glace. Ça fait que, toujours bien, Tit-Renard s'est mis d courir après Gros-Loup pour le poigner, mais il a jamais pu le poigner ${ }^{33}$.

À remarquer que, comme nous l'avons vu dans la tradition amérindienne, l'adversaire du renard peut aussi être dans la tradition franco-canadienne un

${ }^{33}$ Collection Jean-Pierre Pichette, enreg. $n^{\circ} 2791$. Version de M. Bernard Ouimet, 42 ans (né le 21 juin 1941 à Saint-Jacques-le-Mineur, comté de Laprairie, Québec), racontée le 29 octobre 1983 à Easton (rang Meeting-House Road), New-York, États-Unis, où il réside depuis 1953. Il est professeur d'histoire américaine à Cohoes. Conte appris de son grand-père, Médéric Ouimet (1880-1970). On aura noté ici le renversement des rôles, le dupeur étant le loup et non le renard. Bien que, dans le récit qu'il nous a conté immédiatement après celui-ci (voir "La Tinette de beurre», enreg. $n^{\circ} 2792$, dans notre Anthologie de la littérature orale du Canada français, Sudbury, Université de Sudbury, décembre 1985, pp.9-10), les deux mêmes adversaires reprennent leur rôle habituel, il faut éliminer la possibilité d'une méprise involontaire du conteur; il s'agirait au contraire de la vengeance de l'animal trompé, car le conteur nous avait prévenu que «Gros-Loup et Tit-Renard se jouaient des tours». 
ursidé, ainsi qu'il appert dans deux versions acadiennes recueillies à l'île de Shippagan, au Nouveau-Brunswick ${ }^{34}$, et dans un témoignage noté récemment à Plamondon, en Alberta ${ }^{35}$.

\section{Quatrième Partie}

\section{LE POINT TOURNANT}

À ce stade-ci, une question se pose: comment expliquer la présence simultanée dans deux cultures si différentes, l'amérindienne (ici représentée par la version sauteuse de départ et les versions algonquines) et l'européenne (représentée par l'une ou l'autre des versions canadiennes-françaises), d'un même récit de tradition orale? En fait, si l'on écarte comme peu probable la génération spontanée, c'està-dire la création de façon indépendante d'un même récit dans deux cultures différentes ${ }^{36}$, on ne peut

${ }^{34}$ Collection Luc Lacourcière et Félix-Antolne Savard, enreg. $n^{\circ} 914$, «Le Renard et l'ours», version d'Octave Chiasson contée en juillet 1950, et enreg. $n^{\circ} 1262$ de la même collection, "Le Renard et l'ourse", version d'Onias Ferron contée le $1^{\text {er }}$ août 1952.

${ }^{35}$ Lors d'une entrevue préliminaire qu'il nous accordait le 16 avril 1994, M. Noé Saint-Jean disait connaître le conte du petit renard qui fit pêcher un ours avec sa queue.

${ }^{36}$ Voir l'examen de cette "théorie de l'accident" dans l'ouvrage fondamental de Joseph Bédier, Les Fabliaux. Études de littérature populaire et d'histoire littéraire du Moyen Âge (Sixième édition, Paris, Champion, "Bibliothèque de l'École des hautes études", 98; Genève, Slatkine, 1982 [réimpression de l'édition de Paris, 1969, d'après la deuxième édition, 1894], VIII-499 pages), pp. 62-67. Après avoir résumé ainsi lę problème: "Chaque conte ou chaque type de contes aurait pu être inventé et réinventé à nouveau, un nombre indéfini de fois, en des temps et des lieux divers, et les ressemblances que l'on constate entre les contes de divers pays proviendraient de l'identité des procédés créateurs de l'esprit humain", Il affirme: "C'est moins un système organisé qu'une première attitude de l'esprit en présence du problème. C'est une hypothèse qui se présente volontiers à l'esprit de tout apprentl folk-loriste, au début des recherches, et ne résiste pas aux faits» (p. 63). Puis il reconnaît comme "certain que les types généraux, les 
guère envisager que le transfert culturel pour en fournir une explication satisfaisante et rechercher celleci dans les deux seules hypothèses vraisemblables pour les traditions à l'étude: ou bien la tradition canadienne-française a reçu ce récit de la tradition amérindienne, ou bien, et c'est l'inverse, c'est la tradition amérindienne qui l'a reçu de la tradition canadienne-française ${ }^{37}$.

cycles de contes (cycle de la femme obstinée, cycle des ruses de femme) ou les éléments merveilleux des contes (animaux qui parlent, objets magiques) n'appartiennent ni à un pays, ni à [un] temps, et que ces éléments ont pu et dû être mille fois réinventés. Mais ce que nous retrouvons dans les diverses littératures populaires, si nous passons d'un recueil sicilien à un recueil norvégien, ce ne sont pas seulement des types généraux de contes identiques, ce sont les mêmes contes particuliers: c'est, parmi les millions de ruses de femmes qu'on aurait pu imaginer, un nombre restreint de ruses spéciales (la Bourgeoise d'Orléans, les Tresses, le Chevalier d la robe vermeille) et, parmi les millions de contes merveilleux qu'on aurait pu imaginer, un nombre restreint de récits très circonstanciés (la Belle et la Bête, Jean de l'Ours, Cendrillon), c'est-à-dire des contes organisés qui se répètent, ayant l'unité d'une cuvre d'art, la complexité d'une intrigue de roman, portant l'empreinte d'un esprit créateur." De cette observation, il conclut: "Sauf quelques coïncidences négligeables qui ont pu suggérer le même thème très général et très peu circonstancié à deux esprits indépendants, il faut que chaque conte alt été imaginé un certain jour, quelque part, par quelqu'un. Quand? Où? Par qui? La question reste entière, et nul système ne serait viable qui ne pourrait admettre que les contes se propagent par voie d'emprunt" (p. 64).

${ }^{37}$ Cette affirmation, qui paraît éliminer sommairement toute influence des traditions anglophones, anglo-canadienne et anglo-américaine, s'appuie néanmoins sur le catalogue d'Ernest $\mathrm{W}$. Baughman, Type and Motif Index of the Folktales of England and North America (The Hague, Mouton \& Co., [Bloomington], "Indiana University Folklore Series", $20,1966)$, qui ne recense, pour notre récit, qu'une version fragmentaire en anglais (cf. p. 3: elle est du New-Jersey) alors que le catalogue françals en recense 38 versions (Delarue-Tenèze, vol. III) et le catalogue de l'Amérique française en analyse 25 versions (LacourcièreLow, ms. Inédit); comme les contes merveilleux, les contes d'animaux sont sous-représentés en Angleterre et en Amérique anglophone avec seulement 1 I types attestés (pp. 3-5), comparativement à 88 ( 148 avec les sous-types) pour l'Amérique française et à 85 pour la France. 
L'analyse qui précède a bien fait ressortir d'une part les arguments internes qui militent en faveur de la thèse d'une tradition "typiquement amérindienne»: outre les personnages et le décor (des animaux de la forêt canadienne qui sont doués du sens de la parole), on a souligné la présence d'éléments tout à fait caractéristiques des civilisations amérindiennes: le rôle du Fripon mystificateur tenu par le renard d'abord, mais surtout le genre étiologique, récit d'allure mythique relatant l'origine de la queue courte du lynx. D'autre part, un certain nombre d'éléments externes mènent à la même évidence: l'informateur appartient à la tribu des Sauteux du nord de l'Ontario; son récit lui a été transmis, probablement en sauteux ou en anglais, par un ancêtre de sa tribu; il l'a à son tour transmis à un auditeur étranger à sa culture en lui rappelant bien tout le protocole narratif encadrant normalement un mythe; et, enfin, c'est en anglais que ce dernier passage s'est effectué. Voilà pour les éléments vérifiables.

Mais il est d'autres arguments qui viennent ajouter une pression supplémentaire à ces faits internes et externes, et qui relèvent davantage du contexte sociopolitique ou de préjugés bien alimentés par le monde de l'information. À l'heure où les communautés amérindiennes relancent bruyamment leurs revendications séculaires relatives à leurs droits ancestraux, leurs territoires et leur culture ${ }^{38}$, des blancs

${ }^{38}$ Voir le portrait de Georges-Hébert Germain, «Destins. L'Aigle de Matimekosh [Réal McKenzie]", dans l'Actualité (vol. 18, $\mathrm{n}^{\circ} 19,1^{\text {er }}$ décembre 1993, pp. 40-46); en particulier ces commentaires du grand chef des Montagnais: «Tous [les Indiens] s'affirment aujourd'hui haut et fort: sociétés distinctes exigeant réparation, autonomie, respect, en obtenant plus ou moins, soulevant des bouffées de colère et d'envie" (p. 40). Et cet autre: «Il faut maintenant sortir les jeunes de la torpeur. "Ça ne sera possible que lorsqu'on aura acquis notre pleine autonomie 
de bonne volonté s'autoculpabilisent et battent parfois publiquement leur coulpe ${ }^{39}$; d'autres, buvant intégralement et sans discrimination leur discours romantique de peuples vivant en harmonie avec la nature, les citent en exemples et les associent à leurs propres luttes écologistes visant à un plus grand respect de l'environnement naturel ${ }^{40}$.

et qu'on aura un territoire à nous"» (p. 44). Et enfin: "Lorsque les Cris entrent dans le port de New-York ou d'Amsterdam à bord de leur Odeyak et qu'ils se posent à la face du monde en victimes d'un nouvel holocauste et d'une catastrophe écologique commandée par le gouvernement du Québec, ou que Max Gros-Louis parle à la télé française du "génocide" des Indiens québécois, McKenzie applaudit mais s'inquiète, pas sûr du tout que ça peut aider la cause» (p. 46). Martine Turenne, dans un autre article de l'Actualité (Montréal, vol. 17, n० 9,

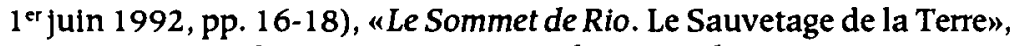
avance une explication: "C'est que les autochtones sont perçus comme des "bons" par les temps qui courent: derniers hommes vivant en "harmonie" avec la nature, ils bénéficient d'un préjugé favorable" (p. 16).

39 Dans un article du même magazine, "Pèlerinage chez le Grand Esprit" (l'Actualité, Montréal, vol. 17, no 17, $1^{\text {er }}$ novembre 1992, pp. 99-101), sous la plume de Luc Chartrand, on peut lire: "Tout de même, en ce $500^{e}$ anniversaire de l'arrivée de Colomb en Amérique, pendant que de nombreux [b]lancs confessent les péchés de découverte de leurs ancêtres, l'endroit [l'Antre de marbre, au lac Albanel] est tout à fait désigné pour un pèlerinage» (pp. 99-100). Parmi ces blancs, pourrait figurer en bonne place le père Robert Vachon, dont le cas rapporté par Christian Rioux, dans un article intitulé «Le Mohawk blanc» paru encore dans l'Actualité (Montréal, vol. 17, no 7, $1^{\text {er }}$ mai 1992, p. 13), illustre notre propos de façon exemplaire: ce «chaud partisan des revendications territoriales amérindiennes [...] a pris la tête d'une délégation de 18 personnes pour rencontrer les représentants des chefs traditionalistes de Kanawake et leur demander la permission d'exercer ses activités sur l'île [de Montréal, car prétend-il: "Nous sommes tous locataires des Mohawks [Agniers] à Montréal"]. Après trois ans "de consultation", les chefs ont finalement exaucé ses vœux en lui demandant toutefois de ne pas ébruiter l'affaire." Toujours d'après l'auteur de l'article, ce père de la congrégation de La Salette avance en outre dans la revue Interculture $\left(n^{\circ} 113\right)$ que «la réalité ellemême de démocratie comme gouvernement par le peuple est venue à l'Occident par le moyen de la nation mohawk [des Agniers] et de la confédération iroquoise».

${ }^{40}$ Le portrait de Pierre-Doris Maltais, ce Québécois né à East-Angus en 
De plus, l'idée d'une irrémédiable dégradation de la tradition orale des blancs, ici des Canadiens français, peut-être encouragée par les travaux des folkloristes eux-mêmes qui ont souvent placé leurs préoccupations dans la recherche des survivances du passé, aurait rejoint le milieu populaire qui constate - conflit de générations oblige - que la mémoire orale des parents, passant de moins en moins à la jeune génération, est reléguée à l'oubli. L'omniprésence du bruit, diffusé par la radio, la télévision, la vidéo et autres techniques de communication sonore, et de la culture savante contemporaine, sorte de prêt-à-porter pour la masse consommatrice, disséminée par l'école, le livre et autres canaux institués, domine et transforme les voix de la tradition orale et laisse peu de place aux modes traditionnels de communication. Si l'on ajoute à ce portrait l'ignorance de l'histoire, en l'occurrence celle du Canada d'avant la Confédération, et le manque de références culturelles qui s'ensuit chez le minoritaire et le convainc finalement qu'il est une anomalie encombrant le groupe majoritaire, on ne s'étonnera guère du résultat. Dans ce contexte, la tradition orale venue des

1937 dont la vie fait l'objet du dernier film documentaire de Jacques Godbout, L'Affaire Norman William, établi par Luc Chartrand, sous le titre "Destins. L'Homme qui se prenait pour d'autres", dans l'Actualité (Montréal, vol. 19, $n^{\circ} 12$, août 1994, pp. 52-57), en fournit un exemple peu banal: ce «sol-disant Indien, porte-parole autoproclamé des peuples métis du monde» (p. 52), fondait en 1976 «la secte écolopacifiste Écoovie», dans la foulée du mouvement écologiste naissant en Europe. «Le culte de l'Indien d'Amérique y est à l'honneur. Les disciples s'installent dans des tipis à Noisy-le-Grand, au bord de la Marne, et le micmac, langue en voie d'extinction des autochtones de la Gaspésie, devient leur langue rituelle. La secte prêche une doctrine écologiste radicale et un tiers-mondisme de bon aloi. [...] La secte vit de manière primitive. "On bouffait mal, on puait et on était tout le temps malades", raconte un ancien membre. Les disciples (environ 160) vendent des aliments dits blologiques et de l'artisanat» (p. 54). 
Amérindiens eux-mêmes aussi bien que les courants idéologiques et politiques récents militent, pour le récit à l'étude, en faveur d'une origine amérindienne et interdit même de penser à une quelconque influence française.

\section{TABLEAU 1}

Liste de noms d'origine française portés par des Amérindiens

(Sauteux)

N.B. Noms extraits de l'Annuaire téléphonique des municipalités de Little Current [Petit-Courant] / Shegulandah, Manitowaning / Wikwemekong / Tehkummah, Mindemoya (île Manitouline)

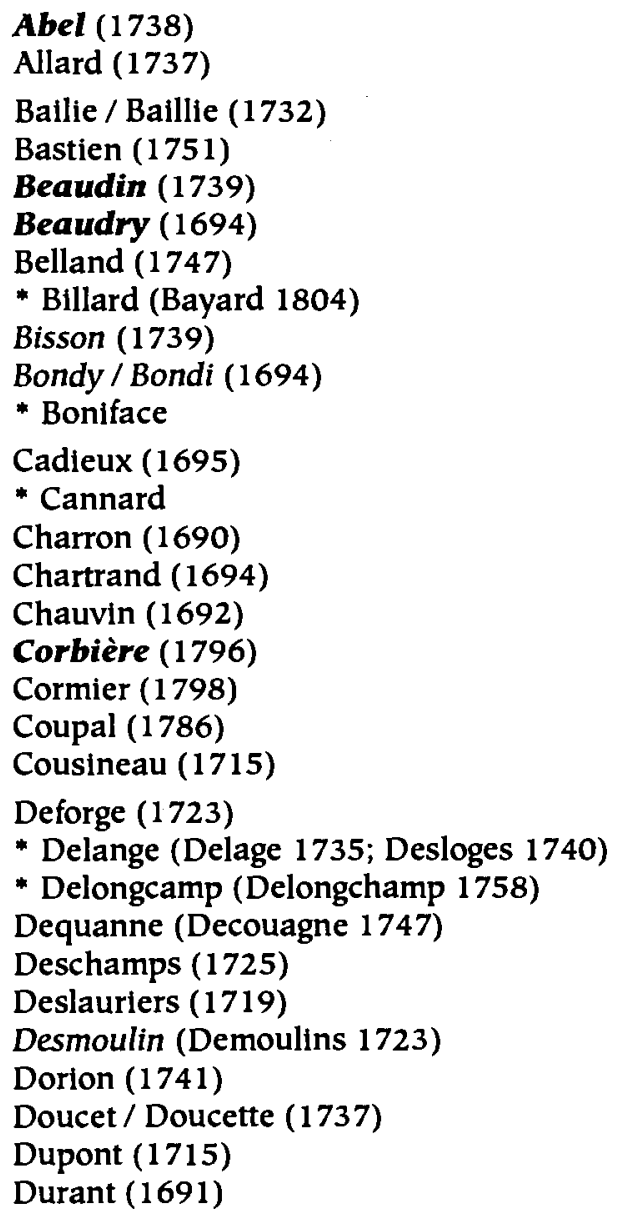


Jean-Pierre Pichette

\section{* Esquimaux}

Félix (1789)

Flamand (1796)

Fortin (1724)

Fournier (1752)

Gagné (1712)

Gagnon (1747)

Gauthier (1688)

Généraux (Généreux 1726)

Geroux / Giroux (1730)

* Girodat

* Glanville

Grevelle (Gravelle 1752)

Guay (1701)

* Guindon

Hertel (1715)

* Ivan

Lacelle (1737)

Lachance (1741)

Lacoste (1715)

LaFleur (1717)

Lafranière (1715)

Lajiness (Lajeunesse 1694)

Lamont (Lemont 1748)

Lamontagen (Lamontagne 1718)

Langlois (1712)

Lapierre (1732)

Laplante (1703)

LaPointe (1703)

Larocque (1748)

Lauzon (1723)

Lavallée (1703)

* Laverty (Laverdure 1724)

LeBlanc (1724)

* LeBroq (Labrèque 1793)

Lecuyer (1705)

Le Papillon (Papillon 1752)

* LeSarge (Lesage 1751; Lestage 1709)

Mallette (1694)

Marechaux (Maréchal 1758)

Marselle (Marcil 1703)

Martell (1737)

Mondoux (1733)

* Morphet (?)

Morrell (1692) 
Mousseau (1688)

* Musquetier

Ouellette (1746)

Ouimette (1744)

Paquette (1690)

* Patreau (Patry 1804; Poitras 1743; Poutré 1737)

Paul (1731)

Pelletier / Peltier (1688)

Perreault (1720)

Prairie (1745)

Prieur (1749)

Proulx (1735)

Pyette (Pillet 1695)

Quesnel (1716)

* Récollet

Rivet (1703)

Roberge (1745)

Rochon (1754)

Rouleau (1724)

* Sabine

Simon (1708)

St Amand (1736)

St Louis (1756)

Thibodeau (1802)

Toulouse (1728)

Trépanier (1747)

Trudeau (Tredo) (Truteau 1688)

Valiquette (1746)

Vallier (Vallié 1703)

* Vautour

Villeneuve (1693)

Vincent (1741)

Viney (Vinet 1690)

15 noms confirmés par Barry Ace pour West Bay le 17 mai 1994. Autres noms connus de lui: Goulais, Lamorandière.

17 noms confirmés par Mary Ann Corbière pour Wikwemekong le 17 mai 1994. Autres noms connus d'elle: Belleau, Boissonneault, Jacko, Lamorandiere, Roque, Syrette, Thibeault.

* 19 noms qui n'ont pu être retrouvés tels quels dans les contrats d'engagement pour l'Ouest.

(1738) Date de la plus ancienne attestation relevée dans les contrats d'engagement pour l'Ouest ( 90 patronymes). 
UN PREMIER INDICE

Pourtant, il nous faut aussi examiner cette hypothèse qui est loin d'être farfelue puisqu'elle se fonde sur la logique et s'accroche en outre à un détail que nous n'avons pas jusqu'ici sollicité. Il s'agit du patronyme du narrateur: Jourdain. Il faut dire que cet indice nous avait tout de suite intrigué et qu'il a ensuite orienté notre recherche dans une tout autre direction. Comme c'est le cas également pour un très grand nombre de Sauteux ${ }^{41}$, ce narrateur porte donc

${ }^{41}$ Un dénombrement sommaire des patronymes français des réserves amérindiennes de l'île Manitouline, celles de West-Bay et de Wikwémékong ainsi que leurs environs, fait d'après les inscriptions contenues dans l' [Annuaire téléphonique de Bell Canada pour la] Municipalité régionale de Sudbury et les environs, février 1994-1995 (Publications Télé-Direct, 1994, pp. 34-43, 49-53), en révèle 109; nous les avons reportés dans le tableau 1 . Deux collègues amérindiens, professeurs à l'Université de Sudbury et originaires de cette île, ont rapidement confirmé qu'une trentaine de ces patronymes sont effectivement portés par des Sauteux. En outre, la très grande majorité de ces 109 patronymes, soit 90 représentant $83 \%$ de ce nombre, ont pu être retrouvés dans les congés de traite octroyés sous le Régime français et les contrats d'engagement pour l'Ouest compilés pour les années 16701821 dont les sources sont les suivantes: É[douard]-Z[otique] Massicotte, "Congés et permis déposés ou enregistrés à Montréal, sous le régime français [ 11 août 1681 - 1 juillet 1737]», dans [PierreGeorges Roy], Rapport de l'archiviste de la province de Québec [dorénavant RAPQ] pour 1921-1922, [Québec], Ls-A. Proulx, 1922, pp. [189]-225; id., "Les Congés de traite sous le régime français au Canada. Congés de traite conservés aux Archives de la province de Québec [6 mai 1739 - 8 juin 1752]", dans RAPQ pour 1922-1923, [Québec], Ls-A. Proulx, 1923, pp. [191]-265; id., "Répertoire des engagements pour l'Ouest conservés dans les archives judiciaires de Montréal [1640-1745]", dans RAPQpour l'année 1929-1930, [Québec], Rédempti Paradis, 1930, pp. [191]-466; id., op. cit. [1746-1752], dans RAPQ pour l'année 1930-1931, [...], 1931, pp.[353]-453; id., op. cit. «[...] (1670-1778)» [1753-1758], dans RAPQpour l'année 1931-1932, [...], 1932, pp. [243]-365; id., op. cit. «[...] (1670-1 778)» [1758-1778], dans RAPQ pour l'année 1932-1933, [...], 1933, pp. [245]-304; id., op. cit. «[...] (1788-1821)» [1788-1797], dans [Antoine Roy], RAPQ pour l'année 1942-1943, [...], 1943, pp. 261-397; id., op. cit. “[...] (17981801)», dans RAPQ pour l'année 1943-1944, [...], 1944, pp. [335- 
un patronyme français ${ }^{42}$. Ce fait, anodin en apparence, témoigne néanmoins d'un métissage séculaire, opéré à grande échelle entre les Français du Canada et les Sauteux, qui remonte à l'époque de la traite des fourrures. Dans le livre qu'elle a consacré à cette tribu, Helen Hornbeck Tanner rappelle à grands traits les premiers contacts entre ces deux cultures.

During this same period [on parle de 1671-1678], French traders arrived at Ojibwa communities and other neighboring Indian villages on Lake Superior to organize the collection and transport of furs to the Montreal market. Initially, the French traders were prohibited from participating in hunting and other community activities because

444]; id., op. cit. «[...] (1802-1804)», dans RAPQ pour l'année 19441945, [...], 1945, pp. [307]-401; id., op. cit. «[...] Louis Chaboillez. ].G. Beek (1805-1821)", dans RAPQ pour l'année 1945-1946, [...], 1946, pp. [225]-340; Jean-Jacques Lefebvre, op. cit. "[...] (1778-1788)», dans RAPQ pour l'année 1946-1947, [...], [1947], pp. [301]-369.

${ }^{42}$ La première mention de ce patronyme apparaît dans la compllation d'É.-Z. Massicotte, «Les Congés de traite sous le régime français au Canada», op. cit., dans le RAPQ pour l'année 1922-1923. À la page 203, on y peut lire l'entrée suivante:

« 5 juin 1743. - Permis du gouverneur de Beauharnois à Nicolas Saint de partir de Montréal avec un canot équipé de six hommes pour se rendre au poste de Détroit. Défense au dit Saint de prendre d'autre route que celle du nord du lac Ontarlo comme aussi de faire aucune traite ou commerce ailleurs qu'au poste de Détroit et ses dépendances. Rôle des engagés du dit canot: Pierre Bertrand dit Toulouse, de Laprairie; François Laliberté et François Lepage, de Lanoraie; Antoine SaintGermain et Joseph Lafleur, de Saint-Michel d'Yamaska; François Jourdain, de Dautray.»

Quelques pages plus loin dans le même article, p. 207, on retrouve un autre contrat d'engagement où apparaît un deuxième Jourdain:

«21 juin 1743. - Permis du gouverneur de Beauharnois au sleur d'Ailleboust de partir de Montréal avec un canot équipé de six hommes pour se rendre au poste de La Baie[-des-Puants, lac Michigan]. Défense au sieur d'Ailleboust de faire aucune traite avec les Sauvages allleurs qu'au poste de La Baie et ses dépendances.

Rôle des engagés du dit canot: François Pilon, de la Pointe-Claire; Pierre Laroche, de Varennes; Jean-Baptiste Lefebvre, de la Pointe-auxTrembles de Québec; Jean Jourdain, Jean Heron et Bernard Dumouchel, de Montréal.» 
they were not related to the Ojibwa. But as time passed, hundreds of young Frenchmen married Ojibwa women and were incorporated into the tribe. Some Ojibwa even created a special clan for their new French kin ${ }^{43}$.

L'union par le sang des Français et des Sauteuses ${ }^{44}$ s'enracinait dans la connaissance et la compréhension mutuelles qu'ils se manifestèrent les uns aux autres à la suite de leur rencontre et de leur fréquentation dans les territoires des Pays d'en haut. Contrairement aux opinions qui circulent encore de nos jours sur la liberté de mœurs des coureurs de bois, ces Français avaient pourtant, auprès des membres de cette tribu, la réputation de se comporter en «bons pères de famille» et de bien s'adapter à leur mode de vie. Ce serait là, explique Helen Tanner, la raison des bonnes relations, entre les Français et ces Amérindiens, qui perdurèrent bien au delà de la Conquête.

Although the British were ultimately victorious on the Pennsylvania front and in the conquest of Canada, Indian people maintained a strong allegiance to the French. Instead of trying to change Indian culture, the French had acquired an understanding of Indian customs. Many had

${ }^{43}$ The Ojibwa, New-York, Philadelphie, Chelsea House Publishers, "Indians of North-America", [1992], (120 pages) p. 37.

${ }^{44}$ Il semble que ce métissage se soit essentiellement, sinon exclusivement, limité à l'union d'hommes blancs, des Français du Canada, à des femmes amérindiennes, des Sauteuses dans le cas présent. Jacques Rousseau écrit: "Qul épouse des Indiens? Des hommes blancs épousent des Indiennes; mais des Blanches n'épousent pas d'Indiens autrefois»; voir "Le Legs amérindien à la culture canadienne-française», Cours de civilisation canadienne-française, Université Laval, Faculté des Lettres, année 1968-1969, second semestre, p. 20. Dans Strangers in Blood, Fur Trade Company Families in Indian Country (Vancouver, University of British Columbia Press, [1980], xxill-255 pages), Jennifer Brown montre comment ces mariages «à la façon du pays» entre des Britanniques, des Écossais surtout, et des Amérindiennes contribuèrent à l'expansion de la traite des fourrures. 
married Ojibwa women and fulfilled their obligations to the tribe by providing for their Indian relatives ${ }^{45}$.

Cette bonne entente s'étendait même aux autorités françaises qui avaient apparemment bien saisi les valeurs amérindiennes. Tanner en donne l'illustration suivante:

French officials had also come to understand the significance of gifts in Indian society, where friends and allies regularly exchanged presents. To the Ojibwa, the greatness of a man was determined by what he gave away, not by what he could save for himself. In a society that believed in sharing, it was considered shameful to hoard one's goods.

On the other hand, the Indians regarded the annual gifts that they received in the name of the French king as a kind of rent. These gifts were considered a suitable exchange for the privilege of using land for forts in Indian country ${ }^{46}$.

Avec le changement de régime, la conception de la valeur des présents se modifia également, mais au détriment des autorités anglaises qui considéraient cette pratique avec un mépris avoué.

If the English king was greater than the French king, they reasoned, English presents should be greater. The Indians were angered when the British actually gave them fewer presents and then behaved with arrogance rather than with respect for Indian leaders.

The first British military officials who arrived in Detroit, where the French command had surrendered in 1760 , did not understand Indian values and their standards for proper behavior. They told the Great Lakes Indians that they should be ashamed for accepting gifts, which the British called "charity". The British also established strict new rules to regulate the fur trade, primarily prohibiting traders from granting the Indians credit. In addition, English

\footnotetext{
45 Tanner, op. cit., p. 48.
}

${ }^{46}$ Ibid., pp. 48-49. 
settlers appeared intent on taking control of the entire region rather than merely trading their goods for furs ${ }^{47}$.

Cette interprétation, qui peut surprendre le lecteur d'aujourd'hui peu familier avec l'histoire de la Nouvelle-France, n'est cependant pas nouvelle et rejoint précisément celle qu'exprimait Benoît Brouillette voilà plus d'un demi-siècle ${ }^{48}$.

Ainsi, les Canadiens, ces Français qui avaient parcouru à peu près tout le territoire de l'Amérique du Nord au cours des voyages d'exploration et de commerce pendant plus de deux siècles ${ }^{49}$, qui avaient fondé de nombreuses colonies le long des routes de navigation et qui avaient joué auprès des Amérindiens "leur rôle traditionnel de conciliateurs, d'autant plus facilement que la race se métissait davantage ${ }^{50} »$, ont certainement pu exercer une grande influence sur la culture orale amérindienne tout comme ils ont pu, à leur tour, être influencés par elle. Mais pour prétendre à une origine canadienne-française, dans le cas qui nous occupe, il faudrait d'abord prouver que

\footnotetext{
${ }^{47}$ Ibid., p. 49.

${ }^{48}$ Volr son livre La Pénétration du continent américain par les Canadiens français. 1763-1846. Traitants, explorateurs, missionnaires, préface de Monsieur l'abbé Lionel Groulx, Montréal, Librairie Granger frères, collection de "l'ACFAS", n० 1, 1939, (242 pages) p. 24: "Le contraste est facile à établir entre le Français ou le Canadien qui recherchait la compagnie et l'amitié des naturels, qui pénétrait hardiment dans le pays, n'hésitait pas à s'allier aux tribus par le lien du marlage, et d'autre part l'Anglais, qui restait distant, qui redoutait les Indiens, les traitait en inférieurs et qui a mis plus d'un siècle avant de se décider à parcourir l'intérieur du pays dont 1 possédait la charte depuis la fin du XVII' siècle."

49 Jacques-Donat Casanova (en collaboration avec Armour Landry) précise que 31 États des États-Unis «ont été découverts, explorés ou colonisés par des Français et des Canadiens français», dans Une Amérique française, [Paris], La Documentation française, et [Québec], L'Éditeur officiel du Québec, 1975, p 121.

${ }^{50}$ Id., op. cit., p. 114.
} 
ce conte était relativement répandu parmi la population canadienne-française.

\section{UN CONTE BIEN CONNU AU CANADA FRANÇAIS}

L'Ontario français, on le sait par le corpus narratif rassemblé par le père Germain Lemieux, a largement contribué à la diffusion du patrimoine oral français en Amérique du Nord. Pourtant, le conte d'animaux semble presque complètement exclu de ce répertoire franco-ontarien. En effet, parmi les 646 contes et autres récits publiés par ce pionnier en 32 tomes sous le titre Les vieux m'ont conté ${ }^{51}$ et dont la moitié environ provient de conteurs franco-ontariens, seulement deux récits sont des contes animaliers ${ }^{52}$. Cette carence remarquable pourrait s'expliquer, non pas tant par l'absence d'une telle tradition chez les conteurs de cette province, mais peut-être surtout par un manque d'intérêt pour les contes d'animaux de la part de notre célèbre folkloriste. Si l'on peut se fier à l'aide-mémoire que ce dernier avait préparé pour ses enquêteurs étudiants, il ne semble pas que ce genre de récits faisait partie de ses préoccupations immédiates puisqu'on n'y trouve aucune entrée suggérant des contes d'animaux dans une énumération d'environ deux cents titres ${ }^{53}$. Le petit nombre de folkloristes

${ }^{51}$ Paris, Maisonneuve et Larose; Montréal, Bellarmin, 1973-1993, 33 tomes incluant un volume d'index.

${ }^{52}$ Vol. 14, no 11 , «La Souris et le charbon», conte type 295, pp. 99-102; vol. 15, $\mathrm{n}^{\circ} 5$, «Voyage de Ti-Jean pour gagner de l'argent», conte type 130, pp. 33-38.

${ }^{53}$ Voir la «Liste de contes destinés à stimuler la mémoire des conteurs», pp. 38-48, faisant suite à la «Liste de chansons folkloriques destinées à stimuler la mémoire des informateurs", pp. 1-37; document polycopié, Université de Sudbury, Institut de folklore, avril 1969 , 48 pages. Dans une entrevue accordée à l'auteur le 26 novembre 1994 (collection Jean-Pierre Pichette, enreg. 3510), le père Germain Lemieux explique l'absence de contes d'animaux dans sa collection par le peu 
qui ont œuvré de façon sporadique sur ce territoire n'ont guère fait mieux puisque Luc Lacourcière et Margaret Low, dans un ouvrage encore inédit, n'en ont relevé que cinq versions pour tout l'Ontario français, mais aucune qui s'apparente de près ou de loin au récit sauteux à l'étude ${ }^{54}$.

C'est là un fait étonnant si l'on considère que ce même catalogue analytique en dénombre vingt-cinq versions, dont quelques-unes en deux ou trois variantes pour un total de trente et une versions, pour l'ensemble de l'Amérique française: treize versions acadiennes ( 2 de l'île-du-Prince-Édouard, $8 \mathrm{du}$ Nouveau-Brunswick et 3 de la Nouvelle-Écosse), onze versions québécoises (des comtés de Beauharnois, Bellechasse, Chicoutimi, Îles-de-la-Madeleine, Kamouraska, Missisquoi, Saint-Maurice) et une version étatsunienne (de la Nouvelle-Angleterre) ont été recueillies ou attestées entre 1942 et $1980^{55}$. Tous ces récits appartiennent aux épisodes types du minicycle de «la Queue perdue et retrouvée» (contes types 2, 2 A, 2 B, 2 C, [AF] $2 C^{*}, 2 \mathrm{D},[\mathrm{AF}] 2 \mathrm{E}$, [AF] 2F), subdivision du très populaire cycle du renard ou de l'animal rusé (contes types 1 à 69) de la catégorie des

d'intérêt qu'il y trouvait - il ne se souvenait pas d'en avoir demandé à ses informateurs - et par le fait "qu'il y avait d'autres choses plus intéressantes ou plus pressantes que ces choses-là», surtout qu'il "aimait mieux enregistrer un grand conte qu'un petit conte" auprès de vieillards qui racontaient peut-être pour la dernière fois de leur vie. ${ }^{54}$ "Le Conte populaire français en Amérique du Nord", tome premier, "Les Contes d'animaux", Sainte-Foy, Presses de l'Université Laval, manuscrit en voie de publication.

${ }^{55} \mathrm{Op}$. cit., conte type 2 et ses subdivisions, «La Queue perdue et retrouvée", 26 p. ms. À ce nombre dont la compilation s'arrête à 1981 , il faut désormais ajouter notre version étatsunienne recueillie dans l'État de New-York et l'attestation franco-albertaine précédemment citées, et peut-être aussi quelques autres versions déposées depuis dans certaines archives de folklore. 
contes d'animaux (contes types 1 à 299) du catalogue international d'Antti Aarne et Stith Thompson ${ }^{56}$. Les versions d'Amérique se répartissent en huit formes dont voici la distribution.

\section{TABLEAU 2}

"La Queue perdue et retrouvée»

Dénombrement des versions françaises d'Amérique d'après le catalogue Lacourclère-Low

conte type titre

\begin{tabular}{|c|c|c|}
\hline ar & [sr] & (nr) \\
\hline 6 & & (6) \\
\hline 1 & & (1) \\
\hline 13 & [7] & (13) \\
\hline 3 & & (3) \\
\hline 18 & [13] & (5) \\
\hline 6 & [2] & (0) \\
\hline 7 & [3] & (0) \\
\hline 8 & [7] & (3) \\
\hline & [25] & (31) \\
\hline
\end{tabular}

8 formes

"La Pêche à la queue"

2 A «La Queue enterrée»

2 B "La Pêche au panier"

$2 \mathrm{C}$ «Sauter par-dessus un feu avec un panier attaché à la queue»"

(AF) 2 C* «La Queue de filasse et les sauts par-dessus le feu"

$2 \mathrm{D}$ «Le Loup est incité à se tourner au vent"

(AF) 2 E "La Queue de fer"

(AF) $2 \mathrm{~F}$ «Perte de la queue de diverses manières"

De ces huit formes, les trois précédées du symbole (AF) sont particulières à notre tradition. Le nombre de versions entre crochets exclut les variantes ou versions différentes d'un même informateur: [sr] signifie donc sans répétition et ar, avec répétition. Ȧ noter qu'une même version peut contenir une, deux, trois et, parfois, jusqu'à quatre de ces formes; c'est pourquoi nous avons ajouté un nombre entre parenthèses qui fait apparaître le nombre réel $(n r)$ de nouvelles versions de cette forme.

On aura remarqué, par la seule énumération des titres, que ce sont les formes des contes types 2 et $2 \mathrm{~B}$ qui se rapprochent le plus du thème à l'étude.

${ }^{56}$ The Types of the Folktale, A Classification and Bibliography, Antti Aarne's Verzeichnis des Märchentypen (FF Communications No. 3) Translated and Enlarged by Stith Thompson, Indiana University, Second Revision, Helsinki, Academia Scientiarum Fennica, «F[olklore] F[ellows] Communications», $\mathrm{n}^{\circ}$ 184, 1961, 588 pages. 
Celles-ci ont en commun un animal stupide, ordinairement le loup, qui, suivant le conseil du renard astucieux, enfonce sa queue par un trou dans la glace afin d'y prendre du poisson; mais, croyant tenir une honnête prise, c'est sa queue gelée qu'il s'arrache $^{57}$. Le type $2 \mathrm{~B}$ diffère cependant par l'ajout d'un panier à la queue de la victime dans lequel l'animal rusé place des roches, sans doute pour assurer le succès de sa mystification ou peut-être pour en accélérer le processus ${ }^{58}$. Ensemble, ces deux types affectent treize narrations différentes recueillies dans quatre provinces canadiennes et dans un État américain. Voilà que cette fourberie du renard, qui inclut bien entendu le type 2 , "la Pêche à la queue», qui est précisément le thème à l'étude, est bien attestée dans l'Amérique française du $\mathrm{XX}^{\mathrm{e}}$ siècle; il est permis de penser que ce récit aurait pu suivre les migrants de la population canadienne-française et se retrouver, avec eux, métissé aux traditions orales amérindiennes. Cette possibilité n'a plus aujourd'hui le caractère inouï qu'elle pouvait avoir au début de ce siècle.

\section{Cinquième partie}

L'INFLUENCE CANADIENNE-FRANÇAISE RECONNUE

C'est à Stith Thompson que nous devons la première démonstration de l'influence de la tradition orale canadienne-française sur celle des populations amérindiennes avec lesquelles elle était venue en contact. Dans la thèse de doctorat qu'il entreprit en 1912 à l'université Harvard et qu'il déposa en 1914 , Thompson se proposait justement d'étudier les

${ }^{57}$ Lacourcière-Low, op. cit., type 2 , "La Pêche à la queue», éléments $A$, A1, (C), C3; type 2 B, "La Pêche au panier», éléments Al, (B), B1. ${ }^{58} \mathrm{Ibid}$, type $2 \mathrm{~B}$, éléments $\mathrm{A}$ et $\mathrm{A} 2$. 
emprunts éventuels et les parallèles de contes européens que les chercheurs avaient retrouvés, accidentellement sans vraiment les rechercher, dans les traditions orales autochtones ${ }^{59}$.

Dans l'introduction de sa thèse, il posait que trois siècles de contacts ininterrompus avec des populations venues d'Europe avaient substantiellement modifié la civilisation amérindienne, comme cela lui paraissait évident au niveau social et économique. Il postulait alors que les transformations avaient débordé ces domaines et qu'elles avaient même influencé son imaginaire, là où les peuples sont ordinairement le plus conservateurs. Il entendait donc en faire la démonstration par l'étude d'une forme caractéristique de cette expression de l'imaginaire amérindien: le conte populaire ${ }^{60}$.

Sa méthode était relativement simple: après avoir sélectionné des contes européens bien établis, écartant ceux dont l'influence directe n'était pas certaine ${ }^{61}$,

\footnotetext{
${ }^{59}$ Sa thèse, intitulée «European Borrowings and Parallels in North American Indian Tales», a été légèrement augmentée et publiée en deux parties quelques années plus tard; c'est la seconde partie, parue sous un titre modifié, qui nous intéresse ici: «European Tales Among the North American Indians», Colorado Springs, Colorado College Publication, «Language Series», vol. II, n³4, 1919, pp. 319-471. Luc Lacourcière, dans son important article «Le Ruban qui rend fort (contetype 590)" du trente-sixième Cahier des Dix (Québec, Éditions des Dix, $n^{\circ} 36,1971$, pp. [235]-297), résume la genèse de la recherche de Thompson et les conclusions convaincantes auxquelles il arriva (pp. 236-243).

${ }^{60}$ Thompson, op. cit., p. 321.

${ }^{61}$ Ibid., p. 321: "Only such stories have been treated here as are fairly complete examples of well-known folk-tales of Old World. No cases where there was reasonable doubt of direct borrowing have been admitted. Hundreds of interesting parallels existing in tales belonging otherwise to native cycles have been left out of consideration, because this whole class of analogues demands separate study."
} 
il les représentait par quelques versions européennes types, puisées principalement chez Cosquin pour la France, les frères Grimm pour l'Allemagne, (et plus tardivement chez Barbeau pour le Canada français, comme on le verra dans sa publication) et les résumait en les décomposant en épisodes. Ensuite, il faisait de même pour toutes les versions amérindiennes connues qu'il disposait par tribu. Finalement, il tirait une conclusion sur l'origine du conte. Il a ainsi soumis à son analyse, en autant de chapitres, vingt-trois contes particuliers, merveilleux et drôlatiques pour la plupart (ch. 1-23), puis il a regroupé dans les cinq derniers chapitres des contes d'animaux (ch. 24), des fables (ch. 25), des récits bibliques (ch. 26), des contes divers (ch. 27) et des références utiles (ch. 28); en tout, quarante-huit récits.

Les résultats de Thompson, qui forment le chapitre final, sont probants. Au terme de sa comparaison, il reconnaissait trois sources principales de diffusion du conte populaire européen en Amérique du Nord: les Français, les Noirs (par l'intermédiaire des Espagnols et des Portugais), et les Espagnols, en prenant soin de préciser cependant l'apport prépondérant des premiers: «By far the greatest contribution has been made by the French, in Canada, and to some extent in Louisiana ${ }^{62}$ \%. Son étude montre aussi que la stabilité des récits au cours des migrations, déjà remarquée sur le vieux continent entre peuples voisins, se maintient en Amérique, bien que passant à des peuples de culture moins avancée, par les conteurs autochtones qui les répètent sans modification notable, même s'ils en perçoivent nettement l'origine

${ }^{62}$ Ibid., p. 456. 


\section{TABLEAU 3}

Contes amérindiens d'origine européenne d'après Stith Thompson «European Tales Among the North American Indians»

$\begin{array}{lll}\text { chapitre / titre du conte* } & \begin{array}{l}\text { conte } \\ \text { type }\end{array} & \begin{array}{c}\text { origine } \\ \text { noire/esp./frse }\end{array} \text { sauteuses }\end{array}$

1. La Bête à sept têtes

2. Jean de l'ours

3. Les Compagnons doués

4. Le Cheval enchanté

5. Le Petit Poucet

6. Le Chat blanc

7. Cendrillon

8. La Véritable Épouse

9. Le Ruban bleu

10. Vérité et mensonge

11. L'Anneau merveilleux

12. Les Pommes magiques

13. Le Cour de l'oiseau magique

14. Le Sauveteur abandonné

15. Les Beaux-frères animaux

16. Faire rire la princesse

17. Surprendre la princesse par une devinette

18. Jean le sot

19. Jean le joueur de tour

20. Le Maître voleur

21. Le Vantard chanceux

22. Le Pari de la colère

23. Jean le fort

24. Contes d'animaux:

Le Renard parrain

La Pêche avec la queue

Le Glouton dans la cave

Le Vol du poisson

La Lune dans le puits prise pour un fromage Les Duellistes peureux Apprendre à craindre l'homme

Les Animaux dans leurs quartlers de nuit
303 et 300

$301 \mathrm{~A}$ et $\mathrm{B}$

$513 \mathrm{~A}$

314

$327 \mathrm{~A}$ et $\mathrm{B}$,

328

$313 \mathrm{~A}$ et $\mathrm{B}$

$510 \mathrm{~A}$

403 B

590

613

561

566

567

569

$552 \mathrm{~A}, 302$

$571(563)$

851

1696,1541

1535

1525 B

1640

1000

$650 \mathrm{~A}$

15

2

41

1

34

104

157

130

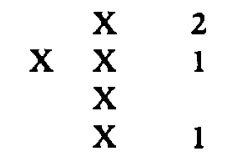

$\begin{array}{lll} & \mathrm{X} & 1 \\ \mathbf{X} & \mathrm{X} & 1 \\ \mathrm{X} & \mathrm{X} & \\ \mathrm{X} & \mathrm{X} & 1 \\ & - & 1\end{array}$

$\mathbf{X} \bar{X}$

$\mathbf{X}$

$\mathrm{X}$

$\begin{array}{ll}\mathrm{X} & 1\end{array}$

$\begin{array}{ll}X & 1\end{array}$

$\mathrm{X}$

$\mathrm{X}$

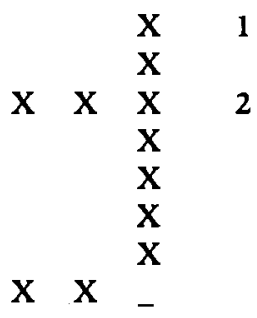

1

2 
Le Loup et les chevreaux 123

Le Bébé de goudron $\quad 175$

Le Lièvre dans les buissons

Le Lièvre prétendant

72

Le Gardien aveuglé

La Division de la récolte 1030

La Course gagnée par ruse 1074 (275)

Faire le mort pour attraper le gibier cf. 33

Les Plumes empruntées

La Course gagnée en s'agrippant à la queue du lion

25. Fables:**

Le Renard et la grue $\quad 60$

Le Coq et le renard $\quad 62$

Le Renard et le corbeau $\quad 57$

La Cigale et la fourmi $280 \mathrm{~A}$

26. Récits bibliques:**

Adam et Ève au paradis

L'Arche de Noé

La Tour de Babel

La Confusion des langues

La Fuite de Joseph en Égypte

Le Passage de la mer Rouge

La Venue de Jésus

27. Contes divers:**

La Vieille et son cochon 2031

Les Souhaits ridicules $750 \mathrm{~A}$

Le Vase ensorcelé

$571 \mathrm{~A}$

28. Autres (références)

* Les titres sont traduits d'après Thompson.

** L'origine européenne de ces récits n'est attribuée à aucun groupe particulier.

étrangère ${ }^{63}$. Constatant encore que certaines tribus qui ont été associées de très près aux Français, particulièrement les Micmacs, les Malécites, les Sauteux, les Indiens de la rivière Thompson et les "Shuswap», ont emprunté un grand nombre de contes européens,

${ }^{63}$ Ibid., p. 456. Thompson dit bien: «At the present time, however, these European tales are felt to be distinctly strangers to Indian folklore, but in spite of that fact to be very welcome strangers.» 
Stith Thompson avance que les répertoires de ces tribus afficheraient vraisemblablement, si les collections étaient complètes, le plus grand nombre de contes étrangers ${ }^{64}$. Il conclut enfin:

We are justified in saying that the great source for the ordinary European folk-tale among the Indians of the United States and Canada is French, and among the Indians of Mexico, Spanish.

\section{Il ajoute toutefois une nuance déconcertante:}

The animal tales here treated are usually of Negro and Spanish origin - all of them ultimately belonging to European tradition ${ }^{65}$.

Si l'on se range volontiers à la première partie de la conclusion générale de Thompson ${ }^{66}$, ce détail final ne manque pas d'étonner: en effet, comment ne pas trouver curieuse cette assertion quand, des dix-huit contes d'animaux analysés par l'auteur, près de la moitié - huit - sont déjà attestés par des variantes françaises d'Europe - c'est le cas précisément du type 2 «La Pêche à la queue» - et d'Amérique et que, plus surprenant encore, la majorité des tribus amérindiennes qui les ont préservés, comme il appert par les cartes préparées par l'auteur lui-même ${ }^{67}$, sont précisément celles qui sont situées dans la sphère

${ }^{64}$ Ibid., pp. 456-457.

${ }^{65}$ Ibid., p. 457.

${ }^{66}$ Luc Lacourcière a démontré que «Le Ruban qui rend fort», qui était «le seul cas auquel il [Thompson] attribu[ait] une origine qui ne soit pas française», "parmi les trente-huit [quarante-huit nous semble plus exact] contes dont il a recherché des parallèles européens pour sa thèse», était également d'origine française. Voir «Le Ruban qui rend fort», op. cit., p. 241 , et les 43 versions canadiennes-françaises qu'il analyse, pp. 265-289.

${ }^{67}$ Thompson, op. cit., a préparé deux cartes géographiques pour des fins comparatives: l'une présente la distribution des contes européens par tribus à l'exclusion des contes d'animaux et des récits bibliques, $p$. 458; l'autre fait de même pour les contes d'animaux européens, p. 459. 
d'influence française, soit le Nord-Est, le Centre et l'Ouest canadien de même que le sud des Grands Lacs et la vallée du Mississippi aux États-Unis? Des influences noire et espagnole semblent difficilement concevables pour ces quatre régions du Nord alors que, au contraire, les influences créole et francolouisianaise apparaissent plus probables dans cette région du Sud. En ce qui concerne cependant les régions situées au nord du Mexique, nous acceptons naturellement et sans réserve l'influence espagnole reconnue par Thompson, sans dénier non plus la possibilité d'une influence noire pour le sud-est des États-Unis.

\section{DÉCOUVERTE DU CONTE POPULAIRE FRANÇASS AU CANADA}

Mais en 1914, tant aux États-Unis qu'au Canada, on connaissait fort peu de contes populaires français $^{68}$ de sorte qu'on ignorait complètement l'impact que cette tradition avait pu avoir en Amérique. Pour

En superposant ces deux cartes, on s'explique mal, sinon par un oubli fâcheux, la réduction, voire l'exclusion, de l'influence française dans la transmission des contes d'animaux que laisse sous-entendre l'expression «usually of Negro and Spanish origin». En ce qui concerne le type 2 «La Pêche à la queue», Thompson se réfère cependant à la version française de Cosquin et, parmi les huit tribus amérindiennes chez qui il a relevé une version, sept habitent la zone d'influence française; cf. pp. 438-443.

${ }^{68}$ Thompson se réfère toutefois à l'ouvrage d'Alcée Fortier, Louisiana Folk-Tales in French Dialect and English Translation (Boston et NewYork, The American Folk-Lore Society, 1895, xi-122 pages) à quatre reprises dans son chapitre consacré aux contes d'animaux (pp. 440441 ); des 41 récits de ce recueil, au moins la moitté est constituée de contes d'animaux. Il cite aussi une fois (p. 454) le livre de William Parker Greenough, Canadian Folk-Life and Folk-Lore (New-York, George H. Richmond, 1897, xil-199 pages), lequel contient, au chapitre quatrième, "Amusements - Contes and Raconteurs", 9 récits d'exagération d'un certain Nazaire, guide de chasse de la région de Québec (pp. 47-60). 
vérifier les conclusions de Thompson, et ainsi mesurer le rôle des Canadiens français dans la propagation du conte européen auprès des Amérindiens, certains anthropologues des États-Unis eurent l'idée d'en rechercher des traces qui appuieraient cette thèse. C'est ainsi que Marius Barbeau, anthropologue au Musée national du Canada depuis 1911 et chargé de l'étude des Amérindiens de l'est du Canada, reçut l'invitation alléchante, de l'un d'entre eux. En 1916, il raconte lui-même cet épisode marquant de sa carrière en commençant sa préface aux «Contes populaires canadiens ${ }^{69} \pitchfork$ :

Il y a près de deux ans, le Dr Franz Boas, de Columbia University, New-York, nous posait la question suivante: "Les Canadiens-français [sic] ont-ils conservé leurs anciennes traditions orales? Y a-t-il encore, en Canada, des anciennes chansons, des contes, des légendes et des croyances populaires?" Il n'était pas facile, à brûlepourpoint, de répondre à cette question ${ }^{70}$.

Barbeau se souvient alors de sa première mission anthropologique, auprès des Hurons en 1911, où il avait rencontré pour la première fois des conteurs, et surtout de leurs contes qui lui avaient fait «une profonde impression»:

Quelle réponse donner? Aucun conte publié. Mais à Lorette, pendant mon relevé ethnographique, je me rappelai que Prudent Sioui et sa femme avaient commencé quelquesuns de ces contes, que j'avais refusés parce qu'ils étaient trop français et d'apparence trop littéraire, entre autres: / La princesse des Sept-Montagnes-Vertes, L'eau de la

${ }^{69} \mathrm{C}$.-Marius Barbeau, «Contes populaires canadiens», dans The Journal of American Folk-Lore, vol. 29, $\mathrm{n}^{\circ} 111$, janvier-mars 1916, $154 \mathrm{p}$. Ce numéro inclut «Un conte de la Beauce» par Évelyn Bolduc, pp. 137. 140; et «Fables, contes et formules» par Gustave Lanctot, pp. 141-151. ${ }^{70}$ Id., p. 1. 
fontaine de Paris, Le corps-sans-âme... Voilà exactement ce que le Dr Boas et la Soc[iété] de Folklore recherchaient ${ }^{71}$.

Sur-le-champ, Barbeau s'engage à lancer de telles recherches, en retour de quoi la «Société de folklore américain» lui offrait «de disposer annuellement d'un numéro entier de sa revue, The Journal of American Folk-Lore» afin de "faciliter la publication périodique de ces matériaux inédits, à mesure qu'on les obtient du peuple ${ }^{72}$ ». On comptait rééditer de cette façon l'expédition organisée par Boas au Mexique et dont la publication des résultats avait débuté sous la direction d'Espinosa.

Quelques mois après cette invitation du Dr Boas, j'allai passer une vacance à Lorette, puis je me rendis à un conventum au Collège de Sainte-Anne, puis à la Beauce. / Première cueillette de contes. À Lorette, avec la famille de Prudent Sioui; à Sainte-Anne, avec Achille Fournier, Thiboutot; à la Beauce, avec le «Père» Patry. Excellente moisson $^{73}$.

En effet:

Quarante contes populaires recueillis en 1914, dans les comtés de Beauce et de Québec, P.Q., démontrèrent que les anciens récits oraux de France se sont conservés intacts. $[. .$.$] / Ces résultats avaient d'ailleurs été prévus par$ M. Boas et certains folkloristes ${ }^{74}$.

Mais pour le jeune chercheur qu'était Marius Barbeau, ce fut une véritable «découverte» qu'il relatera trente ans plus tard dans un cours à l'Université Laval et qu'il considérera alors comme «les

${ }^{71}$ Marius Barbeau, "En quête de connaissances anthropologiques et folkloriques dans l'Amérique du Nord depuis 1911. (Résumé d'un cours donné à la Faculté des Lettres, mars-octobre 1945)», [Québec], Université Laval, Archives de folklore, 1945, (7-82 pages polycopiées) pp. 8-9.

${ }_{72}$ Barbeau, «Contes populaires canadiens», op. cit., p. 2.

${ }^{73}$ Barbeau, «En quête de connaissances [...]», op. cit., p. 9.

${ }^{74}$ Barbeau, «Contes populaires canadiens», op. cit., p. 1. 
débuts, on peut dire officiels, des recherches folkloriques au Canada».

C'était une découverte. Nos conteurs étaient peut-être les meilleurs d'Amérique; il n'y en avait pas eu de meilleurs, autrefois, en France. Ces contes tenaient de la tradition. [...] Pour ma part, j'en étais enchanté. J'ennuyais d'entretiens à ce sujet, mes amis, chez moi et ailleurs ${ }^{75}$.

Ces débordements d'enthousiasme de l'enchanteur Barbeau, devant qui s'ouvrait soudain tout un champ nouveau d'exploration et qui s'émerveillait de constater «que les ressources du folklore canadien sont apparemment inépuisables», allaient bientôt atteindre, par la voie de la revue étatsunienne, l'auteur de cette thèse féconde. Stith Thompson, qui reconnut dans cette cueillette initiale, menée selon les règles scientifiques de l'époque, les premières versions canadiennes-françaises de quatorze des vingt-trois contes particuliers qu'il avait étudiés, voyait ainsi confirmée son hypothèse quant à la source de diffusion des contes populaires européens retrouvés dans les répertoires amérindiens. Aussi, en 1919, au moment de publier le fruit de ses comparaisons, ne manqua-t-il pas d'ajouter, à la fin de son introduction, cette mention: "The conclusions of the dissertation have been greatly strengthened by the publication, in the Journal of American Folk-Lore in 1916 and 1917, of a large body of French Canadian tales $^{76 . "}$

\footnotetext{
${ }^{75}$ Barbeau, «En quête de connaissances [...]», op. cit., p. 10.

${ }^{76}$ Thompson, op. cit., p. 322. En plus des 47 contes de la première série précédemment décrite, il consulta les 31 contes de la deuxième série: C.-Marius Barbeau, "Contes populaires canadiens. Seconde série", dans The Journal of American Folk-Lore, vol. 30, $\mathrm{n}^{\circ} 115$, janvier-mars 191 7, 160 pages; ce numéro inclut «Facéties et contes canadiens» par Victor Morin, pp.141-157.
} 


\section{SIXIÈme PARTIE}

UNE TRADITION FRANÇASE PLUS ANCIENNE

L'hypothèse de Thompson, qui était sérieuse parce que fondée sur la fréquentation des recueils de contes européens, n'en était pas pour autant moins audacieuse. À cette époque, on ne disposait pas encore des précieux instruments de recherche que sont devenus les catalogues nationaux de contes populaires dont Antti Aarne fut l'initiateur; et, même si ce dernier avait publié en 1910 la première version de son catalogue international, Verzeichnis der Märchentypen, cet ouvrage édité en Finlande serait resté inconnu à Thompson jusqu'à la publication de son étude en 1919, puisque sa bibliographie n'en porte pas trace ${ }^{77}$.

De nos jours, des dizaines de pays à travers le monde possèdent de tels outils qui, par l'inventaire détaillé qu'ils dressent de leurs traditions narratives, mettent les chercheurs sur la piste des récits accessibles, tantôt sous la forme d'imprimés ou encore de documents d'archives.

La francophonie européenne compte pour sa part deux de ces catalogues: celui de la Belgique et celui de la France. Dans les Contes populaires wallons, George Laport résume deux versions de notre conte qui furent toutes deux publiées en 1891: la première sous le type «2. La Queue pêcheuse», la seconde sous le type ${ }^{*} 2$.A [sans titre]" qui s'achève par le remplacement de la queue perdue par un postiche en chanvre. Le résumé qu'il en donne est le suivant:

${ }^{77}$ Op. cit., pp. 460-468. D'autre part, il connaissait du même auteur un article de 1908 qui traitait de la comparaison des contes populaires; $\mathrm{cf}$. p. 460 . 
2. La Queue pêcheuse: Le loup n'ayant plus mangé depuis trois jours, le renard l'emmène à la pêche. Il y a de la glace. Le renard fait un trou dans la glace puis conseille au loup de passer la queue dans l'ouverture. L'eau gèle derechef et le loup reste prisonnier. Passe un villageois qui bat le loup. Finalement le loup s'arrache la queue pour se sauver. Il recherche le renard et l'aperçoit de l'autre côté d'un fossé. Le renard crache dans les yeux du loup qui roule dans l'eau ${ }^{78}$.

C'est au troisième tome du catalogue français, inauguré en 1957 par Paul Delarue sous le titre le Conte populaire français et continué par Marie-Louise Tenèze, que se trouve la synthèse des contes d'animaux pour la France et les «pays de langue française d'outre-mer ${ }^{79}$ \%. Ce thème $y$ comporte cinq variantes que nous reportons dans le tableau suivant.

\section{TABLEAU 4}

[«La Queue perdue et retrouvée»]

Dénombrement des versions de France

d'après le catalogue Delarue-Tenèze

conte type

titre

nombre de versions

(nr)

2 "La Pêche à la queue»

23

(+ 2 littéraires)

2 B «La Pêche au panier:

les poissons remplacés" $\quad 15$

2 D "La Queue de chanvre brâlée» 33

[2 $\left.\mathrm{D}^{*}\right]$ «Le Loup brûlé» 2

[2 E] "La Queue soudée»

9

5 formes

Les deux formes entre crochets ont été créées pour la France, mais elles apparaissent au catalogue de l'Amérique française sous d'autres

${ }^{78}$ Op. cit., Helsinki, Academia Scientiarum Fennica, «F[olklore] F[ellows] Communications», $n^{\circ} 101,1932$, p. [25].

${ }_{79}$ Paul Delarue et Marie-Louise Tenèze, Le Conte populaire français, op. cit., pp. 274-284. 
désignations: la forme $\left[2 \mathrm{D}^{*}\right]$ y figure sous la désignation (AF) $2 \mathrm{C}^{*}$ "La Queue de filasse et les sauts par-dessus le feu»; et la forme [2 E] y est désignée (AF) $2 \mathrm{E}$ «La Queue de fer». Le nombre réel (nr) de versions est présenté entre parenthèses.

C'est bien entendu le type 2 qui fait l'objet de notre investigation comme il appert par la liste des éléments qu'en donne le catalogue français:

I. A. Le renard explique au loup comment pêcher du poisson: 1 . à travers un trou dans la glace; 2 . dans un panier (seau) attaché à sa queue; 3. par sa queue; 4. jusqu'à sentir le poids du poisson; 5 . le renard prétend s'y prendre lui-même de cette façon. B (1) Le loup perd sa queue (ou du moins celle-ci est écorchée); B (2) le loup perd les poissons effectivement pêchés; $B$ (3) le loup se noie: 1 . la queue est restée prise dans la glace; 2 . lorsqu'il veut se sauver devant un homme (ou chien); 3 . l'homme lui coupe la queue au ras de la glace dans laquelle la queue est prise.

Les 23 versions orales françaises, toutes recueillies depuis la fin du XIX ${ }^{e}$ siècle, s'inscrivent cependant dans une tradition plusieurs fois séculaire, ainsi que l'attestent deux jalons relayés par l'imprimé. Au XVI ${ }^{\mathrm{e}}$ siècle, un contemporain de Rabelais, Noël du Fail, publie ses Propos rustiques parmi lesquels il énumère les contes que Robin Cheuet récitait à la veillée:

Et ainsi occupés à diverses besongnes, le bon homme Robin (apres avoir imposé silence) commençoit un beau compte du temps que les bestes parloyent (il ny ha pas deux heures): comme le Renard desroboit le poisson aux poissonniers; comme il feit battre le Loup aux Lavandieres, lors quil lapprenoit à pescher; $[\ldots]^{80}$.

\footnotetext{
${ }^{80}$ L'auteur, haut fonctionnaire breton, naquit à Rennes vers 1520 et $y$ mourut en I591; son ouvrage, publié sous le pseudonyme de Maistre Leon Ladulfi, anagramme de Noël du Fail, connut six éditions entre 1548 et 1580 . Voir Conteurs français du XVI siècle, Textes présentés et annotés par Pierre Jourda, [Paris], NRF / Éditions Gallimard, «Bibliothèque de la Pléiade», I956 [1979], p. 620.
} 
Cette simple allusion aux malheurs du loup trompé par le renard repousse l'ancienneté de cette histoire à l'époque de la découverte du Canada par le Malouin Jacques Cartier. Elle a cependant un ancêtre de près de quatre siècles son aîné: il s'agit de la branche III du célèbre Roman de Renart qui aurait été rédigée vers 1178 et qui va bien au delà de l'allusion puisqu'elle en présente la première version complète connue. Étant donné son importance pour notre propos, nous la reproduisons intégralement dans sa traduction en français moderne.

C'était un peu avant Noël, au temps où l'on sale les porcs. Le ciel était clair, étoilé, et le vivier où Ysengrin [le loup] devait pêcher était si gelé qu'on aurait pu danser dessus; les vilains avaient seulement ouvert dans la glace un trou où chaque jour ils menaient boire leurs bêtes. Ils avaient laissé auprès un seau; c'est ld que vint Renart en toute hâte. Il regarda son compère: "Sire, fait-il, approchez par ici! L'endroit est riche en poissons et voici l'engin avec lequel nous pêchons les anguilles, les barbeaux et d'autres poissons bons et beaux. - Frère renart, dit Ysengrin, prenez-le et attachez-le-moi bien à la queue!" Renart lui attache donc de son mieux le seau à la queue. "Frère, dit-il, il vous faut faire sage contenance pour que les poissons viennent. " Alors il alla se coucher près d'un buisson et, le museau allongé entre les pattes, attendit ce qui arriverait à l'autre. Ysengrin est sur la glace et le seau plonge dans le trou; de glaçons il s'emplit à volonté. L'eau en gelant enserre le seau attaché à la queue et la scelle dans la glace. Notre loup songe d̀ se soulever, d tirer le seau d lui. Il essaie de bien des façons, ne sait que faire, s'inquiète. Il commence d̀ appeler Renart; impossible de se cacher maintenant, car l'aube déjà pointait. Renart releva la tête, ouvrit les yeux: "Frère, fait-il, quittez donc le travail; allons-nous-en, beau doux ami; nous avons assez pris de poissons.» Ysengrin lui cria: «Renart, il $y$ en a trop! J'en ai tant pris que je ne sais comment faire." Renart s'est mis d rire et lui a dit sans feindre davantage: "Qui convoite le tout perd le tout." La nuit passe, l'aube perce, le soleil du matin se lève. Les chemins étaient tout 
blancs de neige. Messire Constant des Granges, un vavasseur bien à son aise, qui demeurait près de l'étang, s'était levé, ainsi que sa maisonnée, en menant grande joie. Il saisit un cor, appelle ses chiens, fait seller son cheval; sa maisonnée pousse cris et huées. Renart entend, prend la fuite jusqu'd sa tanière, où il se tapit. Ysengrin resta sur place en bel embarras: de toutes ses forces, il tire, il tire, au risque de se déchirer la peau. Mais, s'il veut partir de là, il lui faudra renoncer d sa queue.

Tandis qu'Ysengrin se secoue, voilà qu'arrive au trot un valet tenant deux lévriers en laisse; il voit le loup tout gelé sur la glace, avec son crâne pelé, et il s'écrie: "Le loup! le loup! au secours! au secours!" Les chasseurs, en l'entendant, sortirent de la maison avec tous leurs chiens. Alors Ysengrin est en détresse; messire Constant venait le dernier sur un cheval au grand galop; il s'écrie: "Laisse, laisse les chiens aller." Les valets découplent les chiens, et les braques s'élancent sur le loup. Ysengrin, tout hérissé, se défend bien et les mord de ses crocs: il n'en peut mais, il aimerait mieux la paix. Messire Constant a tiré l'épée, il s'apprête à bien frapper. Il met pied d terre, et vient au loup sur la glace. Il l'attaque par derrière; il veut le frapper, mais il manque son coup. Il frappe de travers, et messire Constant tombe en arrière: la nuque lui saigne. Il se relève non sans peine. En colere il retourne d l'attaque: quelle terrible guerre! Il crut frapper le loup à la tête, mais c'est ailleurs que porte le coup. L'épée glisse vers la queue, et la coupe rasibus, sans faute. Ysengrin se sent libre, saute de côté et détale, mordant l'un après l'autre les chiens qui le poursuivent et s'accrochent d sa croupe. Il a laissé sa queue en gage: le caur lui crève de rage et de tristesse. Il n'a plus qu'd fuir, il finit par grimper sur un tertre; les chiens le mordent, et lui se défend bien. Une fois montés sur le tertre, les chiens sont recrus de fatigue.

Vers le bois il fuit d grande allure; il y parvient et jure qu'il se vengera de Renart et que jamais il ne l'aimera ${ }^{81}$.

Ainsi, longtemps avant la découverte de l'Amérique par Christophe Colomb, la France connaissait

${ }^{81}$ Le Roman de Renart, extraits, par Jean Frappier et Marc Boyon, Paris, Librairie Larousse, "Classiques Larousse», [1937], pp. 38-39. 
la guerre que se livraient le loup et le renard.

\section{UNE TRADITION EUROPEENNE}

Les versions belges et françaises que nous venons de considérer, et qui sont surtout importantes pour vérifier le point de départ de la chaîne de transmission orale observée en Amérique française, ne composent cependant qu'une infime portion de la tradition quasi universelle dont la dernière édition du catalogue d'Antti Aarne et Stith Thompson, The Types of the Folktale ${ }^{82}$, recensait, il y a maintenant près de trente-cinq ans, 720 versions. Le tableau qui suit, constitué à partir de ces statistiques, donne la répartition des versions dans les 34 pays, ou groupes ethniques, d'Europe, mais aussi d'Asie, où ce type a été retrouvé.

\section{TABLEAU 5}

Dénombrement mondial des versions du type 2 «La Pêche à la queue»

d'après le catalogue international Aarne-Thompson

Europe du Nord

Finlande 156

(Finno-suédois)

Estonie

Livonie

Lettonie

Lituanie

Laponie

26

Suède

Norvège

Danemark

96

Islande

Écosse

Irlande

Total:

${ }^{82}$ Op. cit., pp. 21-22. 
Jean-Pierre Pichette

Europe occidentale

France

Espagne

Pays-Bas

Flandre

Wallonie

Total:

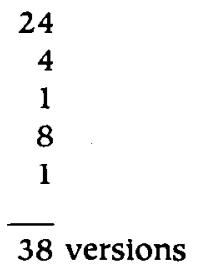

Europe centrale

Allemagne

86

Italie

3

Roumanie

3

Hongrie

Slovénie

10

Serbocroatie

6

Pologne

2

Russie

Grèce

18

Total:

$\overline{138}$ versions

Proche-Orient et Asie

Turquie

2

2

Japon

Total:

(5) versions

Amérique du Nord

Amérique française

Amérique espagnole

Noirs d'Amérique

6

1

1

Total:

8 versions

Grand total:

720 versions

\section{Conchusion}

Au terme de cette longue démonstration, que nous aurions voulue irréfutable, le lecteur conviendra tout de même qu'il serait certainement illusoire de soutenir l'origine amérindienne du conte le Lynx et le renard, car ce serait prétendre en somme que les 
Sauteux du nord de l'Ontario ont inspiré, non seulement les conteurs canadiens-français - et leurs ancêtres français avec, en plus, les auteurs du Roman de Renart qui écrivaient trois siècles avant la découverte de l'Amérique -, mais encore l'ensemble de la tradition européenne qui connaît des récits oraux de l'opposition du renard et du loup beaucoup plus nombreux et des traces littéraires beaucoup plus anciennes recensées jusque chez les fabulistes grecs et latins.

Pour nous, il ne fait done plus aucun doute que cette tradition européenne a été infusée dans le répertoire des conteurs amérindiens par le truchement des conteurs français du Canada à une époque indéterminée sur le territoire actuel de l'Ontario. Les Amérindiens l'ont retenue, l'ont adoptée, sans changement apparent ${ }^{83}$, l'ont reconnue leur, et l'ont à leur tour transmise, enrobée de leur rituel, comme part de leur héritage culturel. C'est bien là ce que Stith Thompson entrevoyait comme possible à la fin de sa thèse sur l'origine des contes populaires européens retrouvés chez les Indiens d'Amérique:

It is possible that this strangeness might wear off if the present state of civilization among the Indians were to persist for centuries, and that the tales might become as much at home on American soil as Hindu tales now seem native to France, Germany, or Norway ${ }^{84}$.

83 Après avoir dénombré 104 contes types empruntés par les Amérindiens au répertolre des 718 types européens contenus dans le catalogue Aarne-Thompson, Stith Thompson peut conclure: «As far as the animal tales are concerned, they appear among the Canadian and southwestern Indians almost without change in the form in which they were learned from the French or the Spaniards." (The Folktale, op. cit., p. 287). Et plus loin il renchérit: «Particularly among the eastern tribes where there has been long contact, the French tales have suffered little change.» (Ibid.).

${ }^{84}$ Thompson, «European Tales Among the North American Indians», 
De plus, les signes qui, dans ce récit, paraissaient à première vue des caractéristiques de son origine amérindienne, sont en réalité des lieux communs attribuables à toutes les cultures européennes chez qui on a recueilli ce conte. Ainsi en est-il de la connaissance de la vie animale, de la coutume de pêcher sur la glace, de la forme étiologique de la narration, du rituel du contage et de sa portée morale, et de la prolifération du récit.

\section{A - Connaissance de la Vie animale}

À vrai dire, il ne semble pas que la connaisance de la vie animale manifestée dans ce récit sauteux soit exclusive au milieu où il fut recueilli; on peut même la qualifier de superficielle tant elle ne dépasse guère les préjugés courants sur ces bêtes, à savoir que le renard est rusé et le loup, stupide. Il n'est même pas sûr non plus que la présence du lynx, à la place du loup ou de l'ours, soit originale si l'on tient compte du fait qu'un glissement linguistique, également possible en français où le lynx s'appelait autrefois loupcervier, a pu se produire dans ses passages anciens et répétés du français à une langue autochtone, le sauteux par exemple, puis de cette langue à une autre jusqu'à la langue de l'enquête, l'anglais dans le cas présent, et enfin à celle de sa diffusion dans l'article étudié, le français. Parmi les 720 versions d'Europe et d'Asie, principalement les 531 de l'Europe du Nord, il se pourrait en outre que l'animal dupé soit aussi un loup-cervier ${ }^{85}$.

op. cit., p. 456.

${ }^{85}$ Nous n'avons pu, pour le vérifier, nous reporter à l'étude de Karle Krohn, Bär (Wolf) und Fuchs. Eine nordische Tiermärchenkette, citée par Marie-Louise Tenèze, op. cit., p. 9. 
B - Coutume DE LA PÊCHE SUR LA GLACE

Coutume connue en Europe, ainsi que le laisse supposer simplement sa présence générale dans les versions belges et françaises, incluant le Roman de Renart, la pratique de la pêche sur la glace, qu'on n'éprouve d'ailleurs jamais le besoin d'expliquer, ne serait donc pas davantage un trait particulier aux versions nord-américaines.

\section{C - Récit étiologique}

On a parfois tendance à considérer le récit étiologique, à cause de sa parenté avec le mythe, comme l'apanage exclusif des sociétés sans écriture. Pourtant les sociétés européennes le connaissent bien, à un point tel que Marie-Louise Tenèze, l'auteur du catalogue des contes d'animaux français a dû exclure, ainsi qu'elle s'en explique en introduction, dans la délimitation de l'objet de son ouvrage, «toute cette belle matière poétique»:

Les récits étiologiques concernant les animaux sont dans les traditions orales françaises si nombreux et si variés qu'il ne peut être question d'en ébaucher ici au passage l'étude $[. .$.$] ni d'en dresser le catalogue: une telle entre-$ prise mérite d'être tentée pour elle-même ${ }^{86}$.

Il en va sans doute de même pour l'Amérique française, bien que les outils de comparaison nous fassent défaut pour le prouver. Les exemples qui en témoignent ne manquent cependant pas: le récit acadien «Pourquoi les loups ont la queue courte», cité in extenso supra, en est un, comme aussi cette

${ }^{86}$ Le Conte populaire français, op. cit., p. 14; voir tout le chapitre «Récits étiologiques», pp. 7-16. 
histoire mensongère intitulée "L'Ours pas de queue», recueillie en Ontario français, mais qui offre une explication complètement différente de la perte de l'appendice ${ }^{87}$.

\section{D - RITUEL ET PORTÉE MORALE}

Si l'offrande de tabac au sage conteur est typique du rituel amérindien, encore que des cadeaux et d'autres privilèges accordés au conteur sont aussi des marques de considération bien attestées dans les traditions européennes, l'attitude de respect que commandent le narrateur et son récit est universelle ${ }^{88}$. Quant à la portée morale des récits animaliers, ce n'est pas une invention nouvelle, les fabulistes grecs et latins ayant reconnu et exploité cette veine il y a

\footnotetext{
${ }^{87}$ Paru dans Héritage de la francophonie canadienne. Traditions orales (sous la direction de Jean-Claude Dupont et Jacques Mathieu, SainteFoy, Les Presses de l'Université Laval, 1986, pp. 260-261), ce récit est composé des contes types 1881 et 1900 . Résumé: Un jeune homme, désireux d'attraper des canards, s'assoit sur une roche dans le fond de l'eau et, tout en fumant sa pipe, attache par la patte, avec une corde retenue à sa ceinture, tous les canards qui se posent sur le lac. Il tape alors des mains pour chasser les volatiles superflus, mais les oiseaux attachés, s'envolant, l'emportent très haut dans les airs. Effrayé, il coupe la corde avec son couteau de poche et tombe dans un arbre creux duquel il est prisonnier. Bientôt arrive un ours noir qui y descend à reculons. Le jeune homme l'attrape par la queue et lui picoche les fesses à l'aide de son couteau. L'ours en sortant entraîne l'homme qui, une fois délivré, lui coupe la queue et peut ainsi rentrer chez lui. "C'est depuis ce temps-là que les ours [n']ont pas de queue.»

${ }^{88}$ Le conteur qui impose le silence, dans la version de Noël du Fail citée supra, en est un premier exemple. Le père Germain Lemieux a déjà abordé indirectement cette question dans les Jongleurs du billochet. Conteurs et contes franco-ontariens (Montréal, Les Éditions Bellarmin; Paris, Maisonneuve et Larose; [Sudbury, La Société historique du Nouvel-Ontario, "Documents historiques" ${ }^{\text {os }}$ 61-62-63], 1972, 134 pages), en traitant de la "personnalité du jongleur», pp. 44-60, et de la "valeur humaine et artistique" de ces "conteurs disparus", pp. 77127.
} 
maintenant deux millénaires, comme le fit aussi au $\mathrm{XVII}^{\mathrm{e}}$ siècle en France le plus célèbre fabuliste français, Jean de La Fontaine.

\section{E - TRANSFERT CULTUREL}

Tout comme la fréquence de ce récit dans les répertoires amérindiens pouvait révéler sa diffusion et son ancienneté, les centaines de versions attestées de l'Europe à l'Asie suffisent à en indiquer l'origine et l'universalité. En somme, l'inclusion de ce récit européen dans la tradition orale amérindienne s'explique d'abord par l'action des conteurs canadiensfrançais qui en ont assuré la transmission et l'infusion. D'autre part, il est certain que le métissage des Français aux Amérindiennes, dont il a été question plus haut, favorisa au plan technique ce transfert. Toutefois, ce facteur déterminant s'insérait déjà dans un phénomène plus global: le choc culturel sans précédent qui allait bouleverser de fond en comble les valeurs des peuples autochtones. Jacques Rousseau et George W. Brown résument bien l'ampleur de ce phénomène.

Pour les Indiens du Nord-Est de l'Amérique, le choc culturel s'est fait sentir violemment dans trois secteurs d'importance vitale. En premier lieu, le secteur idéologique: leurs croyances religieuses et leur système tribal ont été ébranlés jusqu'à la racine, au point de saper l'autorité des chefs et des sorciers. En deuxième lieu, le secteur économique: la traite des pelleteries a provoqué une révolution sociale marquant chaque trait de la vie quotidienne, semant la discorde parmi les tribus pour aboutir aux "guerres de la fourrure». Enfin, le secteur politique: les rivalités entre les empires français et anglais ont sans cesse entraîné les Indiens dans les luttes des envahisseurs cherchant la suprématie militaire en Amérique. Dans chacun de ces domaines, les conséquences furent beaucoup 
plus profondes et tragiques pour les Indiens que pour les Blancs $^{89}$.

Pourtant, les Anglais, qui contribuèrent aussi à ce choc, eurent, sur l'oralité amérindienne, un impact nettement plus limité. Il serait même nul s'il faut en croire l'opinion de Stith Thompson.

The negative side of this picture is quite as interesting as the positive. Unless our collectors have been very negligent, we seem to be forced to the conclusion that that group which was to become dominant in Canada and the United States, and which carried the British tradition to this country, has contributed nothing to the folklore of the American Indians.

Et l'explication qu'il se hâte de proposer rejoint celle que nous avons exprimée.

Many things help to explain this, but the most important fact is that the British never fraternized with the natives, and particularly did not intermarry with them as did Frenchmen or Spaniards ${ }^{90}$.

En plus des influences française et espagnole, la tradition orale amérindienne a également été perméable à l'influence africaine, notamment par la présence d'une population noire dans le sud-est des

${ }^{89}$ «Les Indiens du Nord-Est de l'Amérique», dans le Dictionnaire biographique du Canada, op. cit., vol. 1, p. 8.

${ }^{90}$ The Folktale, op. cit., p. 286. Dans la conclusion de l'Observance des conseils du maître, Monographie internationale du conte type $910 \mathrm{~B}$ (Helsinki, Academia Scientiarum Fennica, «Folklore Fellows Communications» $n^{\circ} 250$; Sainte-Foy, Les Presses de l'Université Laval, "Les Archives de folklore» $n^{\circ} 25,1991$, p. 574), nous faisions semblable observation: "Il faut signaler ici l'absence d'une tradition angloaméricaine au Canada et aux États-Unis. Déjà anémiée en Angleterre, la connaissance du type $910 \mathrm{~B}$ ne pouvait guère survivre en Amérique; il ne semble pas non plus que les Anglo-Américains aient, à ce point de vue, profité du voisinage français et espagnol, comme l'ont fait les Amérindiens, en dépit du fait que seize des dix-huit versions recueillies aux Etats-Unis proviennent de leurs concitoyens d'origine latine.» 
États-Unis. Découverte par Alcée Fortier ${ }^{91}$, démontrée par Stith Thompson ${ }^{92}$, confirmée par Élisabeth Brandon $^{93}$, cette interprétation sera revue et corrigée par Alan Dundes, dans son article «African Tales Among the North American Indians», qui avancera même que le cycle du lièvre fripon est, lui aussi, d'origine africaine.

Therefore since the rabbit is not a trickster figure outside the southeast in American Indian folklore and since there can be no question that African narrative elements were introduced into American Indian tales, one can plausibly argue that the rabbit trickster figure so popular in American Negro tradition is African, not American Indian ${ }^{94}$.

Quoi qu'il en soit, il est évident que les Canadiens français et les Sauteux partagent aujourd'hui, en ce qui concerne "le Lynx et le renard», une tradition depuis longtemps commune. "Gens de parole» aujourd'hui comme au temps de la Nouvelle-France, ces Français d'Amérique ont su maîtriser la communication orale, celle qui, en raison de l'analphabétisme dominant, était le partage du plus grand nombre ${ }^{95}$.

\footnotetext{
${ }^{11}$ Louisiana Folk-Tales, op. cit., p. [ix].

92 "European Tales Among the North American Indians", op. cit., p. 426, 449, pp. 456-457.

${ }^{93}$ "Le Conte français en Louisiane», dans Internationaler Kongreß der Volkserzählungsforscher in Kiel und Kopenhagen (19.8. - 29.8.1959), Berlin, Walter de Gruyter, 1961, pp. 34-36.

${ }^{94}$ Southern Folklore Quarterly, Gainesville, University of Florida, vol. 29, no 3, septembre 1965, p. 218.

${ }^{95}$ Le philosophe Emmanuel Kant ne s'en étonnerait pas, lui qui, dans son Anthropologie du point de vue pragmatique, précisait la différence des caractères des Français et des Anglais. "La nation française, écrivait-il, se caractérise entre toutes les autres par le goût de la conversation [...] par un besoin immédiat de son goût de s'ouvrir aux autres.» Par contre, la nation anglaise, qui «se voit sans conteste supérleure [...], est fort insociable». Cette opposition, qui paraît même dans leur langue, «tient sans doute à la différence de situation, continentale pour l'une, insulaire pour l'autre». Il résume ainsi leur rivalité: «appréhension d'un côté, haine de l'autre; deux modes de leur incompa-
} 
Cette simple démonstration, faite au niveau régional, illustre l'étendue des transferts culturels entre les Français d'Amérique et les Amérindiens ${ }^{96}$, et montre l'avantage qu'il y aurait à réviser les idées reçues, particulièrement au chapitre des traditions orales où la propriété intellectuelle est plus difficilement démontrable, en partie à cause des préjugés qui abondent. Notre intention n'était pas ici d'établir la propriété intellectuelle de ce récit, mais bien de reconnaître comment il s'était retrouvé chez les Sauteux du nord de l'Ontario. Ceux-ci, se l'appropriant, n'ont fait, en dernière analyse, qu'appliquer la maxime attribuée à Molière: «Je prends mon bien où je le trouve." Dès lors, seront-ils capables de reconnaître que, partiellement de sang français eux aussi, ils sont, comme les Canadiens français, les continuateurs de cette longue tradition?

\section{ÉpILGue}

\section{LE CHAINON MANQUANT}

Durant la préparation de cette étude, qui s'est échelonnée sur plusieurs mois, nous avons en outre sondé la tradition orale franco-ontarienne. Mais celle-ci

tibilité, dont le premier vise à la conservation de soi-même, le second à la domination de l'autre, et, sinon, à son anéantissement" (CEuvres philosophiques, III. Les derniers écrits, Édition publiée sous la direction de Ferdinand Alquié, Paris, Gallimard, 1980, pp. 1124-1127).

${ }_{96}$ Dans notre article, «La Diffusion du patrimoine oral des Français d'Amérique" (récemment publié dans Langue, espace, société. Les Variétés du français en Amérique du Nord, sous la direction de Claude Poirier avec la collaboration de Aurélien Boivin, Cécyle Trépanier et Claude Verreault, Sainte-Foy, Les Presses de l'Université Laval, "Culture française d'Amérique», 1994, pp. [127]-143), nous reconnaissions ce fait qui se dégageait naturellement des travaux de Marius Barbeau et de Luc Lacourcière. Nous ne pensions pas alors pouvoir à notre tour en faire la démonstration. 
semblait, contrairement à celle des Sauteux, avoir perdu la mémoire de ce conte d'animaux. Pourtant, elle n'allait pas longtemps demeurer en reste. Voilà que, au moment de rendre cet article, notre collègue Donald Deschênes trouvait, parmi les travaux de ses étudiants qu'il avait eu l'amitié d'intéresser à notre recherche, l'excellente version que voici, la première relevée en Ontario français.

Le conte de l'ours et du renard

Mon grand-père Charles, quand j'étais jeune, il nous posait des fois la question:

- Sais-tu comment ça se fait que les ours ont la queue courte?

Évidemment, nous autres, on disait:

- Je le sais pas.

- Bien, je vas te conter un conte, te conter une histoire.

Ça fait bien bien bien longtemps de ça. Il y a bien bien longtemps. Les ours, ils avaient une belle grand-queue comme les renards puis comme les loups, une belle grand-queue là, en circulaire là là, une belle grand-queue qui frisait là. Ils avaient une belle queue, f'avait pas de bon sens, toute bien touffue là, puis leur queue, je te dis qu'ils étaient fiers de ça; c'était comme un coq avec sa grand-queue courbée. Ils faisaient leurs farauds avec ça.

Les ours étaient aussi [de] mauvais caractère dans ce tempslà qu'd cette heure, puis quand le loup ou bien donc le renard, ils avaient des proies là, qu'ils étaient après manger ça la, l'ours arrivait là puis il disait:

- Donne-moi ça!

Et puis, eux autres, ils aimaient pas ça. Mais l'ours était beaucoup plus fort qu'eux autres. S'ils lui donnaient pas, il te leur fourrait une claque puis fa allait revoler, tu comprends bien, comme un paquet de poils au vent. Puis là, l'ours, bien il dévorait leur proie. Puis eux autres, bien, ça les mettait en fureur, tu peux croire. 
Ça fait qu'une bonne fois, ils ont dit:

- Quelque bonne fois, on va l'avoir. On va se revenger.

Ça fait qu'une bonne fois, le renard - ll y avalt un pêcheur qui passait avec son cabarouet ld. Il y avait un poisson qui était tombé en bas - puis le renard était après le manger, ce poisson-ld. Puis l'ours arrive puis, comme de coutume, en grognant puis en montrant la dent:

- Donne-moi ça, ce poisson-ld!

Le renard, il dit:

- Tu peux bien l'avoir, il dit, je peux en avoir tant que je veux, du poisson de même.

L'ours, il dit:

- Comment ça, que tu peux en avoir tant que tu veux?

- Bien, il dit, oui, il dit, j'ai trouvé un truc ld, il dit. À cette heure ld, il dit, je peux pêcher puis je poigne tout le poisson que je veux.

Bien, c'était le commencement de l'hiver, à peu près comme ce temps-ci de l'année là. L'ours, il dit:

- Comment [est-] ce [que] tu fais ça?

- Bien, il dit, je fais un trou dans la glace ld puis je me mets la queue dans la glace ld. Puis, il dit, le poisson me mord après la queue puis, il dit, quand il y a pas mal de poissons qui sont poignés après ma queue, je sacre un coup ld puis, il dit, je sors le poisson: je prends tout le poisson que je veux. Tu peux bien l'avoir, celui-ld, il dit, je vais aller m'en poigner d'autres. Ça me dérange pas!

L'ours, il dit:

- Penses-tu que je pourrais en poigner?

- Bien, il dit, le renard, avec une queue comme tu as ld? Bien, il dit, tu pourrais en poigner bien plus que moi!

- Bien, l'ours, il dit, tu vas me montrer comment faire.

- Bien, le renard, il dit, c'est bon.

Ils ont trouvé un trou dans la glace là. Il dit: 
- Tu vas t'assir ici, là là, le péteux sur le bord du trou la, puis, il dit, tu vas laisser ta grand-queue dans l'eau la. Puis, là, tu vas voir: d̀ un moment donné ld, fa va commencer à tirer un peu. Mais, il dit, tire pas tout de suite, il dit, laissele mordre comme il faut, il dit, et tu vas en poigner plus.

Comme de fait, l'ours s'assit sur le bord de la glace, mes vieux. Bien, c'était fret sur le passe-bine, tu peux le croire, mais il avait sa queue dans l'eau ld. Ah! que c'était fret! Puis il endurait, il endurait. À un moment donné, il se déplace un peu:

- Aie! il dit, fa commence d tirer.

Le renard, il dit:

- Grouille pas, grouille pas, il dit, grouille pas, il dit, f̧a commence d mordre, il dit. Attends qu'il y en ait bien gros.

L'ours endurait puis endurait. Puis, après que la glace a été bien gelée ld:

- Ah, il dit, ça tire pas mal.

Le renard, il dit:

- Donne un bon coup, il dit, tu vas en poigner un paquet, il dit, tu vas en poigner une trâlée.

L'ours se recule un petit brin, mes vieux, puis il te sacre un coup, puis il se coupe la queue dret da ras la glace. Puis c'est depuis ce temps-là que les ours ont la queue courte!

Puis ça, c'est la fin de l'histoire ${ }^{97}$.

97 DFUS, collection Julie Perron, enreg. $n^{\circ} 2$. Version de Camille Perron, 66 ans, enseignant à la retraite originaire d'Astorville, contée le 5 janvier 1995 au même endroit; récit appris il y a longtemps de son grand-père, Charles Perron. On notera le caractère fortement étiologique de ce conte dont la narration prend place après la question posée par le conteur. L'allusion au poisson trouvé par le renard appartient au type 1 «Le Vol du poisson». 
Remerciements. En terminant, nous désirons remercier les personnes suivantes qui nous ont apporté leur amical concours dans la préparation de cette étude: d'abord et surtout, Pascal Guillemette, l'auteur de l'article qui l'a déclenchée; nos collègues de l'Université de Sudbury, Roger Spielman, Mary-Ann Corbière, Barry Ace, Serge Saint-Pierre et Yvan Morals; Donald Deschênes et Germain Lemieux du Centre francoontarien de folklore; Margaret Low, René Dionne, Gaétan Gervals et Fernand Ouellet. Tous ont lu et commenté cet article, ou nous ont suggéré des pistes utiles. Enfin, Julie Perron mérite un merci tout particulier puisqu'elle a recueilli la première version orale francoontarienne de ce conte animalier; c'est probablement l'un des derniers enregistrements du conteur Camille Perron dont le décès est survenu le 6 juin 1995. 